\title{
Use cases and scenarios of 5G integrated satellite-terrestrial networks for enhanced mobile broadband: The SaT5G approach
}

\author{
Konstantinos Liolis ${ }^{1}$ (D) | Alexander Geurtz ${ }^{1}$ | Ray Sperber ${ }^{1}$ | Detlef Schulz ${ }^{1}$ | \\ Simon Watts ${ }^{2}$ | Georgia Poziopoulou ${ }^{2}$ | Barry Evans ${ }^{3}$ | Ning Wang ${ }^{3}$ | Oriol Vidal ${ }^{4}$ | \\ Boris Tiomela Jou ${ }^{4}$ | Michael Fitch ${ }^{5}$ | Salva Diaz Sendra ${ }^{5}$ | Pouria Sayyad Khodashenas ${ }^{6}$ | \\ Nicolas Chuberre ${ }^{7}$
}

\footnotetext{
${ }^{1}$ SES S.A., Betzdorf, Luxembourg

${ }^{2}$ Avanti Communications Group plc, London, UK

${ }^{3}$ University of Surrey, Surrey, UK

${ }^{4}$ Airbus Defence \& Space SAS, Toulouse, France

${ }^{5}$ British Telecommunications PLC, London, UK

${ }^{6}$ i2CAT Foundation, Barcelona, Spain

${ }^{7}$ Thales Alenia Space France, Toulouse, France Correspondence

Konstantinos Liolis, SES S.A, Château de Betzdorf, L-6815 Betzdorf, Luxembourg. Email: konstantinos.liolis@ses.com
}

Funding information

European Union's Horizon 2020 research and innovation program, Grant/Award Number: 761413

\section{Summary}

This paper presents initial results available from the European Commission Horizon 2020 5G Public Private Partnership Phase 2 project "SaT5G" (Satellite and Terrestrial Network for 5G). ${ }^{1}$ After describing the concept, objectives, challenges, and research pillars addressed by the SaT5G project, this paper elaborates on the selected use cases and scenarios for satellite communications positioning in the $5 \mathrm{G}$ usage scenario of enhanced mobile broadband.

\section{KEYWORDS}

5G Satellite-Terrestrial Integrated Networks, eMBB (enhanced mobile broadband), use cases, scenarios, backhauling, multicasting, caching, offloading

\section{1 | INTRODUCTION}

$5 \mathrm{G}$ is the next generation of communication technology that much of the world is moving to. By supporting a world in which "anyone and anything will be connected at anytime and anywhere", $5 \mathrm{G}$ is expected to enable new applications in various domains, including media and entertainment, health, automotive, transport, and industry. The advanced communications of $5 \mathrm{G}$ are expected to bring enhanced mobile broadband (eMBB), ultrareliable and low latency communications, and massive machine-type communications (mMTC), which correspond to the $5 \mathrm{G}$ usage scenarios defined by ITU-R ${ }^{3}$ for International Mobile Telecommunications (IMT) for 2020 and beyond (IMT-2020) (see Figure 1).

The next-generation network of $5 \mathrm{G}$ has been described as having significantly more capacity and higher user data rates than today's capabilities, so as to meet the growing demands of users. In addition, an important goal of $5 \mathrm{G}$ is to provide increased resilience, continuity, and much higher resource efficiency including a significant decrease in energy consumption. Finally, security and privacy will need to be ensured to protect users and the important amounts of data that will be carried across the network. The $5 \mathrm{G}$ Key Performance Indicators (KPIs), such as 1,000x capacity, 10 to 100x higher typical user data rate, better/increased/ubiquitous coverage, service creation in minutes, and end-to-end (E2E) latency of $<1 \mathrm{~ms}$, are summarized in 5G Public Private Partnership (PPP) and European Commission. ${ }^{4,5}$ Note that these 5G PPP KPIs listed above are not expected to be met all at the same time. No single technology will meet all of these needs, and not all of these characteristics will be required for every $5 \mathrm{G}$ application. On the contrary, as the European Commission and other governments around the world have correctly recognized, to be successful and meet user demands, the $5 \mathrm{G}$ infrastructure will be an ecosystem of networked networks, utilizing multiple different and complementary technologies.

To this end, many organizations, including the European Commission, ${ }^{6}$ recognize that satellite networks will be an element of the $5 \mathrm{G}$ infrastructure. Among others, the role of satellites in $5 \mathrm{G}$ has been studied in the EU Technology Platform NetWorld 2020 SatCom WG ${ }^{7}$ as well as 


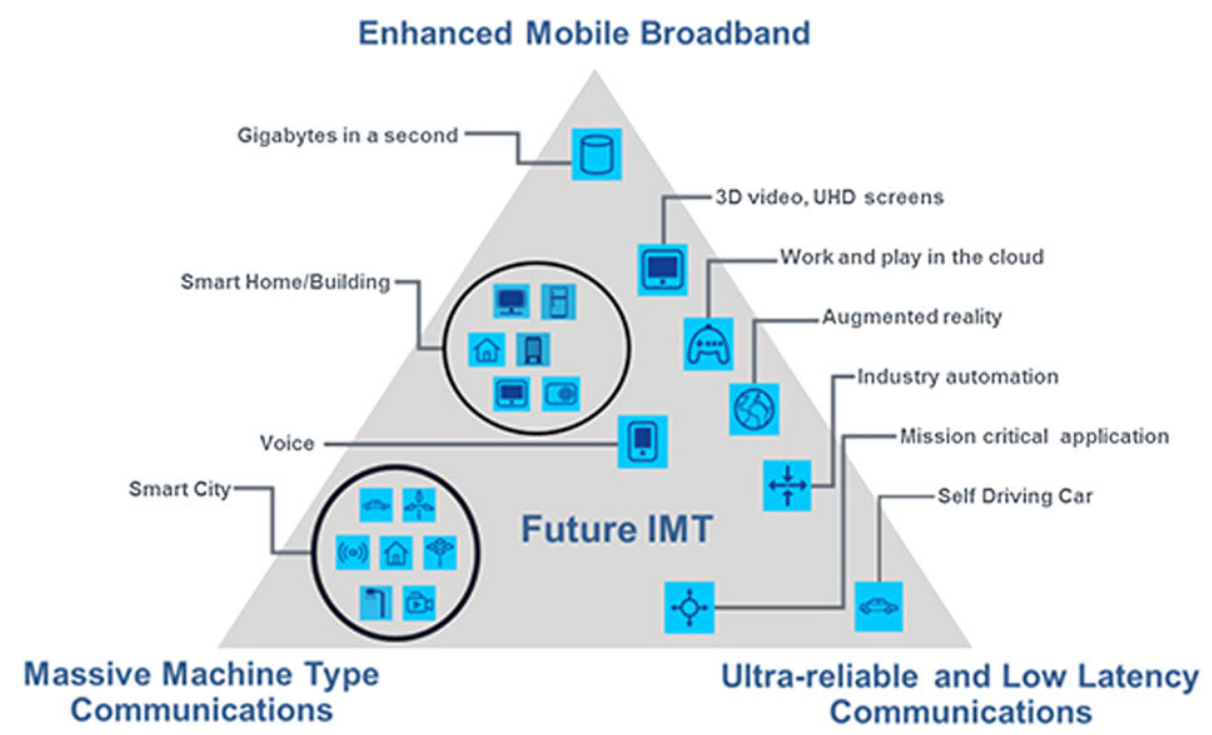

FIGURE 1 Usage scenarios of IMT for 2020 and beyond (source: ITU-R ${ }^{3}$ ) [Colour figure can be viewed at wileyonlinelibrary.com]

in relevant R\&D projects, such as SPECSI, ${ }^{8}$ MENDHOSA ${ }^{9}$ and INSTINCT, ${ }^{10},{ }^{11}$ CloudSat, ${ }^{12}$ SANSA,${ }^{13}$ VITAL, ${ }^{14}$ RIFE,$~^{15}$ and SCORSESE. ${ }^{16}$ Moreover, the EMEA Satellite Operators Association (ESOA) has published a 5G White Paper on the satellite communication (SatCom) services' role as an integral part of the $5 \mathrm{G}$ ecosystem. ${ }^{17}$ The consensus and wider agreement on what satellite brings toward achieving the $5 \mathrm{G}$ KPIs are as follows:

- Ubiquity: Satellite provides high-speed capacity across the globe using the following enablers: capacity in-fill inside geographic gaps, overspill to satellite when terrestrial links are over capacity, general global wide coverage, back-up/resilience for network fall-back, and especially communication during emergency.

- Mobility: Satellite is the only readily available technology capable of providing connectivity anywhere on the ground, in sea, or air for moving platforms, such as airplanes, ships, and trains.

- Broadcast (simultaneity): Satellite can efficiently deliver rich multimedia and other content across multiple sites simultaneously by using broadcast and multicast streams with information centric networking and content caching for local distribution.

- Security: Satellite networks can provide efficient solutions for secure, highly reliable, rapid, and resilient deployment in challenging communication scenarios, such as emergency response.

One may note that the features above are mainly due to satellite's ability to serve coverages that are wider than most other communications technologies. Not only can satellites provide ubiquitous, anytime coverage, but they can provide cost-effective coverage to many areas of the globe, which might otherwise go unserved. Satellite can offer complementary connectivity options, seamless user experience, and provide important benefits.

Satellites are the only means to provide truly ubiquitous geographic coverage and mobility. This feature is critical to the successful deployment and operation of $5 \mathrm{G}$ :

- providing wide coverage to complement and extend dense terrestrial cells;

- complementing connectivity for mobile nodes (ships, airplanes, vehicles, and trains);

- offloading a temporarily congested network;

- providing backhauling services to fixed or moving base stations; and

- providing emergency response/disaster recovery communications.

Satellites are inherently well suited to broadcast or multicast one-to-many transmission links, usually over long distances and large areas to multiple distribution hubs such as radio access points. Satellites can deliver very high data rate services in broadcast/multicast mode (eg, data broadband connectivity and IP-enabled video distribution via satellite) as well as in unicast mode. Satellites today deliver very high data (>100 Mbps-1 Gbps) in broadcast mode to outdoor radio access points for the following:

- high capacity 2-way broadband services for complementary coverage to fixed or terrestrial wireless networks outside major urban/suburban areas; 
- direct delivery of linear/nonlinear video transmissions TV/IPTV services to in-building fixed customers; and

- interconnection via 3G/4G/4G+/Radio Local Area Network wireless access networks (for in-home/in-building distribution) for service delivery to in-building "mobile" users.

The diversity of designs among satellite systems also contributes to their utility in support of the $5 \mathrm{G}$ ecosystem. Increasingly, satellite systems deliver greater capabilities at lower costs by leveraging the particular characteristics of each system's global reach:

- A single GEO (geostationary Earth orbit) satellite can provide communication downlinks over wide areas, such as entire countries or continents, including to rural areas with no terrestrial connections. Further, a single GEO satellite can deliver high-bandwidth, high-reliability services to a large number of connected devices, whether fixed or in-motion, simultaneously across a wide geographic region.

- Constellations of nongeostationary satellites can deliver high-capacity services to localized areas with low latency that some applications require. Such satellites are operating already (eg, SES's O3b MEO [medium Earth orbit] HTS [high throughput satellite] constellation), with more constellations being planned for the 2020 timeframe, in time to participate fully in the $5 \mathrm{G}$ ecosystem.

In addition, with the requirement to have very low delay services, there is an incentive to place services in the edge nodes resulting in less stringent requirements on the delay in the backhaul. This opens up further opportunities for satellite networks as one of the most reliable and secure forms of backhauling.

Leveraging these varied strengths of SatCom expands the range, capacity, and capabilities of $5 \mathrm{G}$ systems.

Figure 2 below illustrates the role of SatCom in the $5 \mathrm{G}$ ecosystem.

The remainder of this paper is structured as follows: Section 2 introduces the SaT5G project concept, objectives, and research activities. Section 3 elaborates on the selected satellite use cases for eMBB. Section 4 defines the specific scenarios for the selected satellite use cases, and Section 5 concludes the paper.

\section{I SAT5G CONCEPT, OBJECTIVES, AND RESEARCH PILLARS}

SaT5G ${ }^{1}$ is a European Commission H2020 5G PPP Phase 2 project, kicked-off in June 2017 with 30-month duration, whose vision is to develop cost effective "plug and play" SatCom solutions for 5G to enable telecom operators and service providers to accelerate 5G deployment in all geographies and at the same time create new and growing market opportunities for SatCom industry stakeholders.

SaT5G focuses primarily on backhaul via satellite.

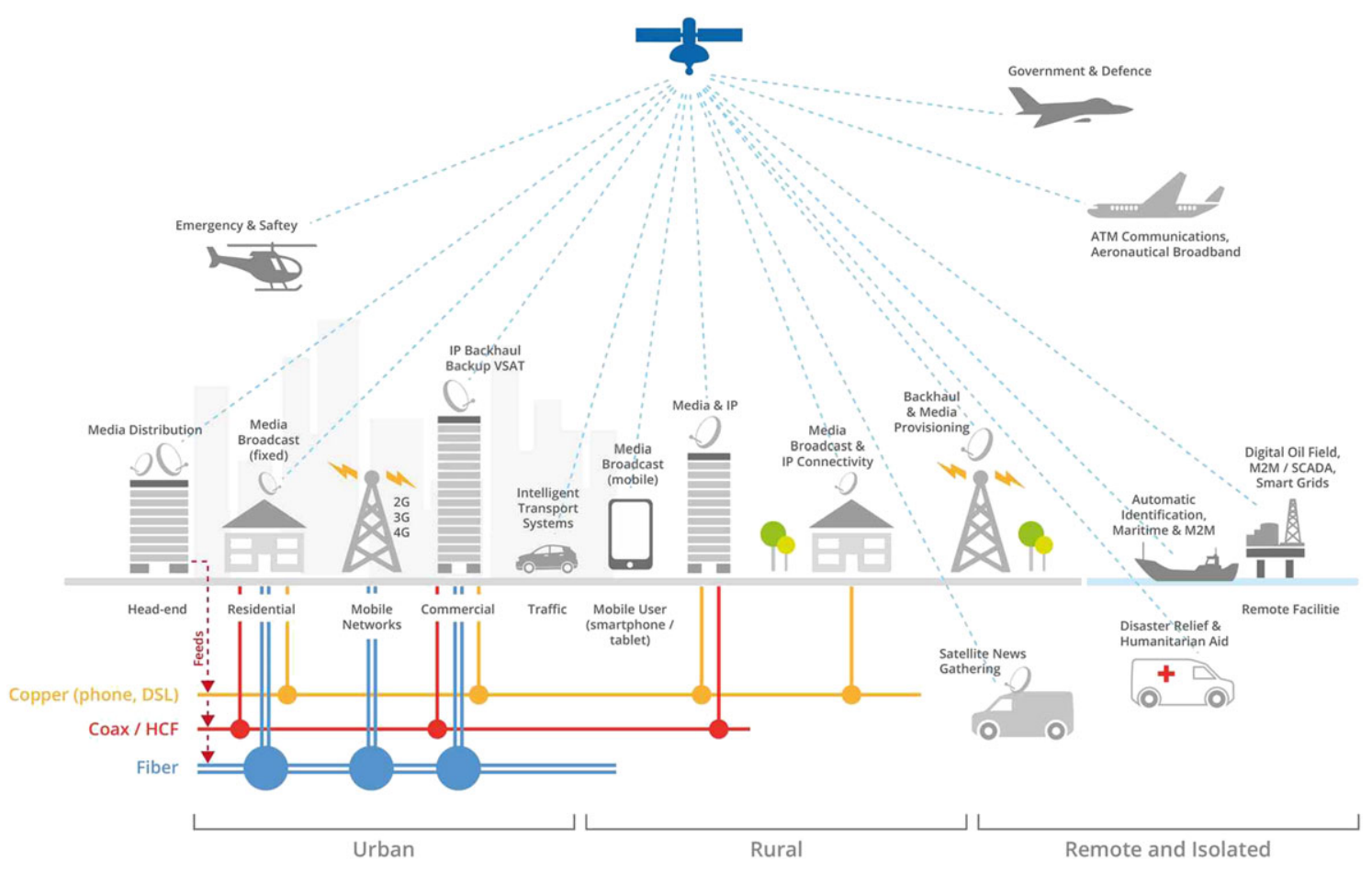

FIGURE 2 Satellite network role in 5G (source: ESOA ${ }^{17}$ ) [Colour figure can be viewed at wileyonlinelibrary.com] 
The 6 principal SaT5G project objectives are to:

- leverage relevant ongoing $5 \mathrm{G}$ and satellite research activities to assess and define solutions integrating satellite into the $5 \mathrm{G}$ network architecture;

- develop the commercial value propositions for satellite-based network solutions for 5G;

- define and develop key technical enablers for the identified research challenges;

- validate key technical enablers in a lab test environment;

- demonstrate selected features and use cases with in-orbit GEO and MEO HTS satellites; and

- contribute to the standardization at ETSI (European Telecommunications Standards Institute) and 3GPP (Third-Generation Partnership Project) of the features enabling the integration of SatCom solutions in $5 \mathrm{G}$.

With the identified satellite strengths and based on the anticipated market needs, SaT5G focuses on the eMBB Usage Scenario for 5G. Based on the analysis results obtained from relevant ESA (European Space Agency) Advanced Research in Telecommunications Systems projects, such as SPECSI ${ }^{8}$ and MENDHOSA, ${ }^{9}$ the broadband and broadcast services will have the highest revenue in 2025 and thus form the primary SaT5G target markets. Furthermore, from the mobile operator's viewpoint for the inclusion of satellite support in the early $5 \mathrm{G}$ roll out, congested backhaul and offloading high bandwidth video download have been found to be the major drivers. These operator drivers also fall under the $5 \mathrm{G}$ usage scenario of eMBB. Therefore, SaT5G addresses specifically the eMBB usage scenario toward "broadband access everywhere." This is not to say that SatCom may not benefit other 5G usage scenarios, such as the mMTC, for instance, but only that the indicated 5G usage scenario of eMBB appears most attractive for SatCom.

The technical challenges that need to be addressed for the realization of cost-effective "plug and play" SatCom solutions for 5G include the following:

- virtualization of SatCom network functions to ensure compatibility with the 5G SDN (software defined networking)/network function virtualization architecture;

- developing the enablers for an integrated 5G-SatCom virtual and physical resource orchestration and service management;

- developing link aggregation scheme for small cell connectivity mitigating quality of service and latency imbalance between satellite and cellular access;

- leveraging 5G features/technologies in SatCom;

- optimizing/harmonizing key management and authentication methods between cellular and satellite access technologies; and

- optimal integration of the multicast benefits in $5 \mathrm{G}$ services for both content delivery and VNF (virtual network function) distribution.

To rise to these challenges, the SaT5G concept comprises 6 research pillars (RPs) and 3 horizontals as shown in Figure 3 . The horizontals address global issues cutting across the whole project while the RPs have been selected to address the deeper research enablers relating to the identified challenges and needed to flow into prototypes to be used in the validations and demonstrations. Our key contribution in SaT5G is the incorporation of the integrated test bed that will validate and demonstrate the mature SaT5G solutions.

The SaT5G horizontals selected are as follows:

\section{Business and operations}

Satellite systems are evolving, with greater capacity (in the order of Tbps speed) with lower cost per bit and better connectivity which makes the integration of satellite with terrestrial mobile networks a compelling commercial proposition. The business aim is to improve customer experience and value, with lower incremental capital cost than fixed network upgrades. The near $100 \%$ coverage provided by the high vantage point of a satellite, and the characteristic distance-independent cost of adding users, is increasingly desirable for mobile network operators (MNOs) for capacity and coverage.

The business opportunities fall into 2 categories:

- Offloading and caching in the fixed network: provision of alternative or overlay paths for (a) connecting data centers to 5G core network gateways where there are no terrestrial or economically viable paths and (b) connecting the core network to semicentralized or centralized virtualized eNodeB processing centers.

- Backhaul: Provision of alternative or overlay paths for backhaul connections to 5G cells in all layers (macro, micro, etc). Opportunities are (a) backhaul to cells in remote areas that are installed to achieve a certain coverage obligation, for example, to satisfy license or contractual requirements, and where terrestrial link is not economically viable, (b) cells that need fast deployment and cannot wait for terrestrial provisioning, (c) deployment of cells for temporary events, and (d) deployment of small mobile cells mounted on public transport, on high-end cars, or on emergency service vehicles. 


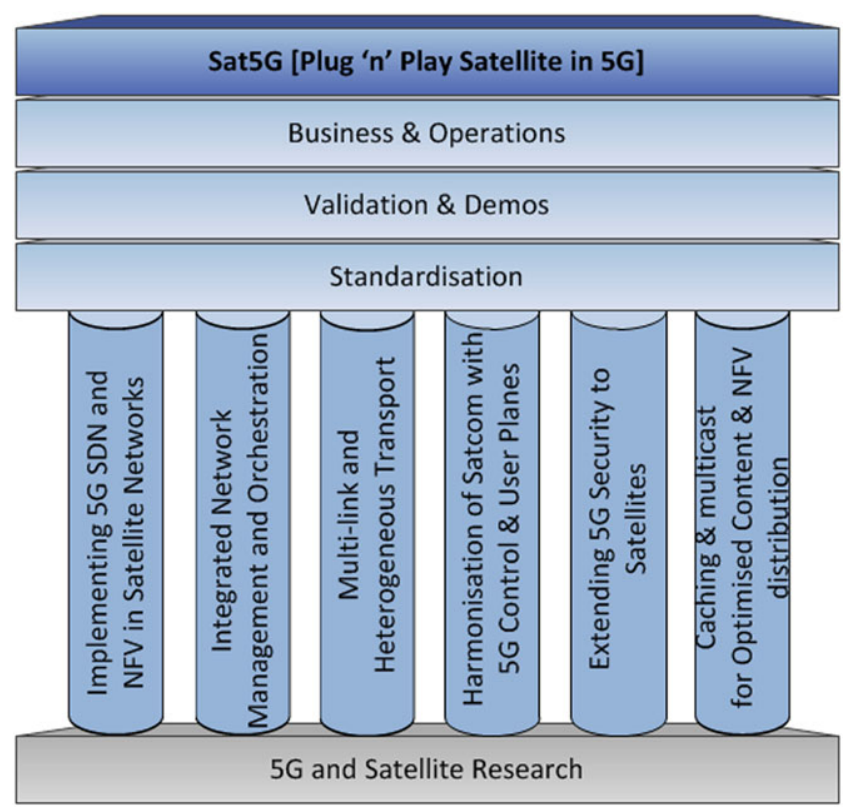

FIGURE 3 SaT5G concept [Colour figure can be viewed at wileyonlinelibrary.com]

SaT5G will demonstrate that by integration of SatCom, new business opportunities are created to engage major stakeholders. SaT5G will propose new business models including the new value chains and diverse actors as well as show how they will come together to operate such a system. SatCom will expand the opportunity for mobile operators by enabling fast roll out of services in a way that has not been possible for $3 \mathrm{G} / 4 \mathrm{G}$.

Standards

Standardization is the key to future equipment interworking and to reduce the eventual costs of equipment as it opens up manufacturing scalability. Third-Generation Partnership Project has established a pathway to 5G standardization onto which SaT5G will build on. SaT5G partners have already been contributing into 3GPP and ETSI groups as a satellite working group. ${ }^{18}$ The inclusion of the satellite elements into 3 GPP and ETSI standards is a major component of the SaT5G project, and partners are committed to contributing and influencing standardization meetings. Outputs from SaT5G are fed into the relevant groups as they mature and feedback from standards will continuously advise the program of activities.

With the development of 5G, SatCom systems can leverage open standards to reduce the deployment and operational cost of terminals as well as other satellite network infrastructure equipment (eg, gateway) and, very importantly, to facilitate a tighter operational integration ("plug and play" approach) into a heterogeneous 5G "network of networks" under common network management. The ongoing standardization efforts around $5 \mathrm{G}$ thus provide a unique opportunity to insert the satellite-specific "hooks" as may be required.

\section{Validation and demos}

SaT5G will develop the key interfaces that allow the integration of the virtualized satellite elements into a terrestrial 5G test bed. The terrestrial test bed is currently virtualized, and thus, SaT5G will develop virtualized satellite gateway and terminal virtualization integration in the test bed. Other elements such as the management and orchestrator component and the multiaccess edge computing (MEC) component for use in caching and multicast will similarly be developed prior to integration and demonstration trials with in-orbit GEO and MEO HTS satellites operating at frequency bands above $10 \mathrm{GHz}$.

The 6 RPs chosen along with their scope and benefits are presented in Table 1 below.

The SaT5G RPs along with the SaT5G use cases (see Section 3) are illustrated in Figure 4 below.

\section{3 | SELECTED SATELLITE USE CASES}

\section{1 | Selection methodology}

By definition, a $5 \mathrm{G}$ use case is a particular case of how the $5 \mathrm{G}$ system is used, whereas a satellite use case in $5 \mathrm{G}$ is a particular case of how the SatCom system is integrated within the $5 \mathrm{G}$ ecosystem. The selected use cases elaborated in this section correspond to specific satellite use cases for eMBB, which have been selected to be further investigated in the SaT5G project. 
TABLE 1 SaT5G research pillars scope and benefits for the 5G ecosystem stakeholders

\begin{tabular}{|c|c|c|}
\hline Research Pillar (RP) & Scope & Benefits \\
\hline $\begin{array}{l}\text { Research pillar I: implementation of 5G SDN } \\
\text { and network function virtualization (NFV) } \\
\text { across satellite networks }\end{array}$ & $\begin{array}{l}\text { Virtualize SatCom network functions to share the same } \\
\text { virtualized core as cellular network functions, ensure } \\
\text { compatibility with the SDN/NFV architecture, and support } \\
\text { network slicing. }\end{array}$ & $\begin{array}{l}\text { CAPEX (capital expenditure) reduction and } \\
\text { flexible service provisioning }\end{array}$ \\
\hline $\begin{array}{l}\text { Research pillar II: integrated network } \\
\text { management and orchestration }\end{array}$ & $\begin{array}{l}\text { Enable integrated 5G-SatCom virtual and physical resource } \\
\text { orchestration and service management. }\end{array}$ & $\begin{array}{l}\text { OPEX (operational expenditure) reduction } \\
\text { through harmonized network } \\
\text { management between } 5 \mathrm{G} \text { and SatCom }\end{array}$ \\
\hline $\begin{array}{l}\text { Research pillar IV }{ }^{\mathrm{a}} \text { : harmonization of } \\
\text { SatCom with } 5 \mathrm{G} \text { control and user plane }\end{array}$ & $\begin{array}{l}\text { Leverage } 5 \mathrm{G} \text { features in satellite radio access network and } \\
\text { foster the integration with other network technologies. }\end{array}$ & $\begin{array}{l}\text { CAPEX and OPEX reduction (especially } \\
\text { development/maintenance effort for } \\
\text { future SatCom) }\end{array}$ \\
\hline $\begin{array}{l}\text { Research pillar V: extending } 5 \mathrm{G} \text { security to } \\
\text { satellite }\end{array}$ & $\begin{array}{l}\text { Provide an efficient key management and authentication } \\
\text { method and harmonize authentication and authorization } \\
\text { between terrestrial and satellite technologies. }\end{array}$ & $\begin{array}{l}\text { Trust enforcement in the E2E 5G network } \\
\text { including satellite element }\end{array}$ \\
\hline
\end{tabular}

${ }^{a}$ With respect to research pillar IV and in line with SaT5G Consortium, ${ }^{18}$ note that SaT5G investigates both of the following features and pursues relevant standardization activities: (a) a 5G core network-friendly non-3GPP satellite access technology, which is pursued within the ETSI TC-SES SCN Working Group, and (b) a satellite-friendly new radio (NR) protocols and access technology architecture, which is pursued within the 3GPP RAN Working Group.

With focus on the eMBB usage scenario for $5 \mathrm{G}$ and by following the methodology illustrated in Figure 5 below, SaT5G selected 4 satellite use cases for eMBB to concentrate its efforts on.

Specifically, we consolidated a "global" list of satellite use cases in $5 \mathrm{G}$ by review, gap analysis and taking into account satellite use cases in $5 \mathrm{G}$ identified at 3GPP domain (eg, 3GPP TR 22.891, ${ }^{19}$ 3GPP SA1, "SMARTER Technical Reports, ${ }^{20}$ 3GPP TR 22.863 V14.1.0 (2016-09), ${ }^{21}$ 3GPP TR 22.864 V14.1.0 (2016-09), ${ }^{22}$ and 3GPP TR 38.811 ${ }^{23}$ ), at SatCom domain (eg, ESOA 5G White Paper ${ }^{17}$ ), as well as by other relevant EU and ESA R\&D projects (eg, SPECSI, ${ }^{8}$ MENDHOSA ${ }^{9}$ and INSTINCT, ${ }^{10},{ }^{11}$ CloudSat, ${ }^{12}$ SANSA, ${ }^{13}$ VITAL, ${ }^{14}$ RIFE, ${ }^{15}$ SCORSESE, ${ }^{16}$ and High Throughput Digital Broadcasting Satellite Systems ${ }^{24}$ ).

Furthermore, by filtering the consolidated "global" list of satellite use cases in $5 \mathrm{G}$ for eMBB, we came up with a subset of satellite use cases for eMBB which was analyzed in detail based on their relevance to the associated core 5G PPP KPIs, the relevant 3GPP SMARTER use case

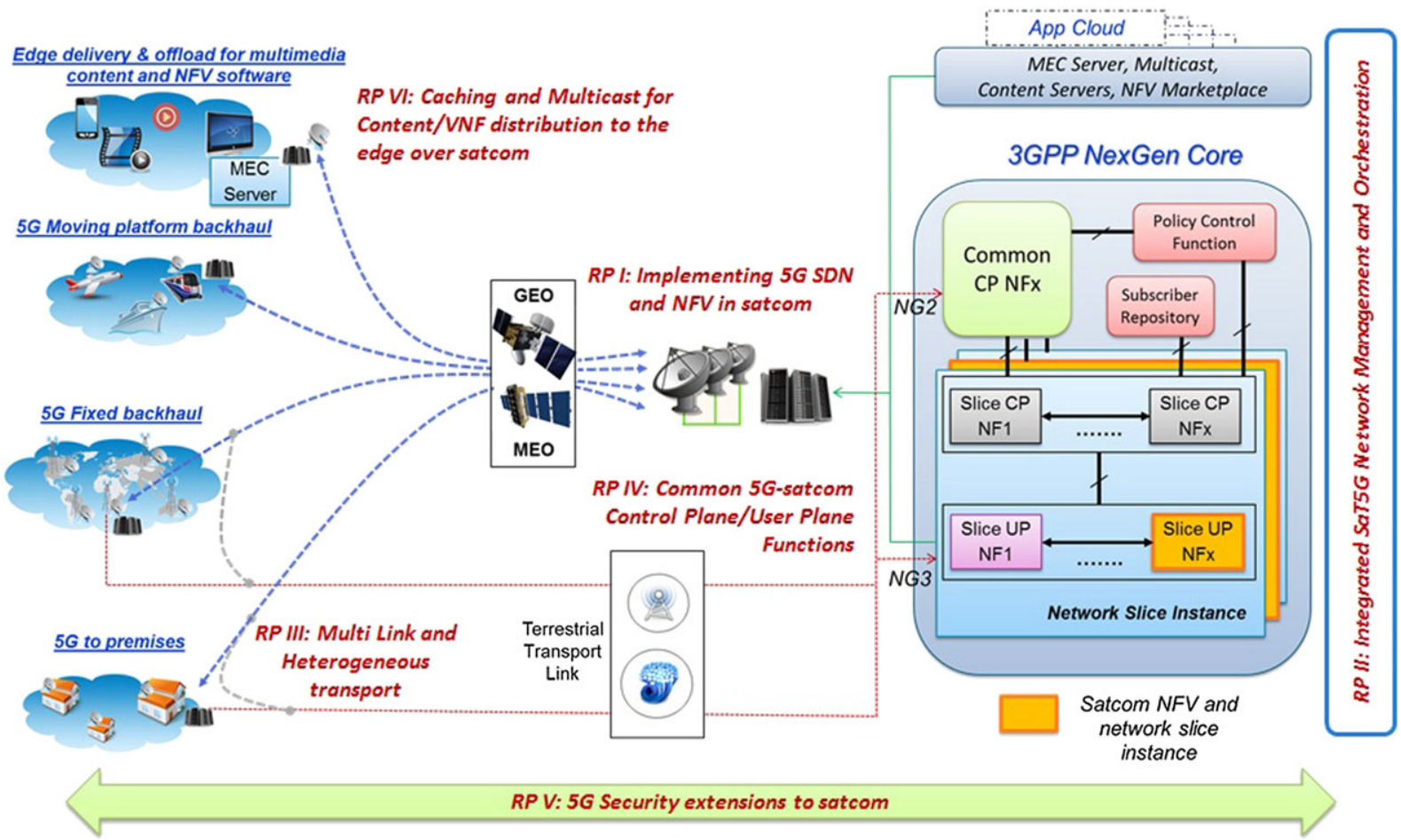

FIGURE 4 SaT5G use cases and research pillars [Colour figure can be viewed at wileyonlinelibrary.com] 


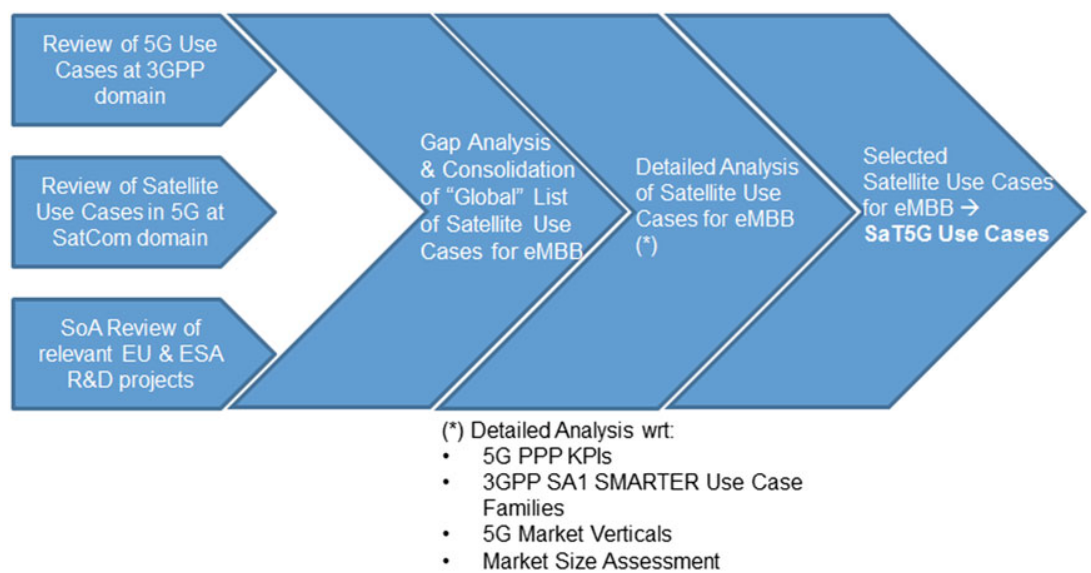

FIGURE 5 Selection methodology for SaT5G use cases [Colour figure can be viewed at wileyonlinelibrary.com]

families, the 5G market verticals, as well as their relevant market size. Based on this detailed analysis, the satellite use cases for eMBB selected for further investigation in the SaT5G project correspond to the SaT5G use cases, which are presented in this section.

Due to space limitations, the detailed analysis of the satellite use cases for eMBB is not reported here. To this end, the interested readership is referred to SaT5G Consortium. ${ }^{25}$

\subsection{Selected satellite use cases for eMBB}

By following the methodology illustrated in Figure 5 above, SaT5G selected 4 satellite use cases for eMBB to concentrate its efforts on the following (see Table 2):

- edge delivery and offload of multimedia content and MEC VNF software, through multicast and caching to optimize the operation and dimensioning of the $5 \mathrm{G}$ network infrastructure;

- 5G fixed backhaul, to provide 5G service especially in areas where it is difficult or not possible to deploy terrestrial communications;

- $5 \mathrm{G}$ to premises, to provide 5G service into home/office premises in underserved areas via hybrid terrestrial-satellite broadband connections; and

- $5 \mathrm{G}$ moving platform backhaul, to support 5G service on board moving platforms, such as aircraft, vessels, and trains.

Figure 6 below illustrates the SaT5G use cases and how they will be integrated into a 5G network.

\section{3 | Relevance to satellite "sweet spots" in 5G}

As can be deduced from Table 2 above, each SaT5G use case corresponds to 1 of the 4 satellite use case categories (SUCCS) in 5G identified by ESOA, ${ }^{17}$ or else referred to as satellite "sweet spots" in 5G (see Figure 7). Each of these 4 broad categories of satellite use cases in $5 \mathrm{G}$ has distinct connectivity characteristics, which are elaborated below:

- Trunking and head-end feed: This SUCC in 5G addresses high speed trunking of video, loT (Internet of Things), and other data to a central site, with further terrestrial distribution to local cell sites, for instance neighboring villages, as shown in Figure 8 below. A very high speed

TABLE 2 SaT5G use cases: Selected satellite use cases for eMBB

\section{SaT5G Use Case}

SaT5G use case 1: "edge delivery and offload for Prov multimedia content and MEC VNF software"

SaT5G use case 2: "5G fixed backhaul"

SaT5G use case 3: "5G to premises"
Description

Providing efficient multicast/broadcast delivery to network edges for content such as live broadcasts, ad-hoc broadcast/multicast streams, group communications, and MEC VNF update distribution

Broadband connectivity where it is difficult or not (yet) possible to deploy terrestrial connections to towers, for example, maritime services, coverage on lakes, islands, mountains, rural areas, isolated areas, or other areas that are best or only covered by satellites; across a wide geographic region

Connectivity complementing terrestrial networks, such as broadband connectivity to home/office small cell in underserved areas in combination with terrestrial wireless or wireline
Broadband connectivity to platforms on the move, such as airplanes or vessels on the move 


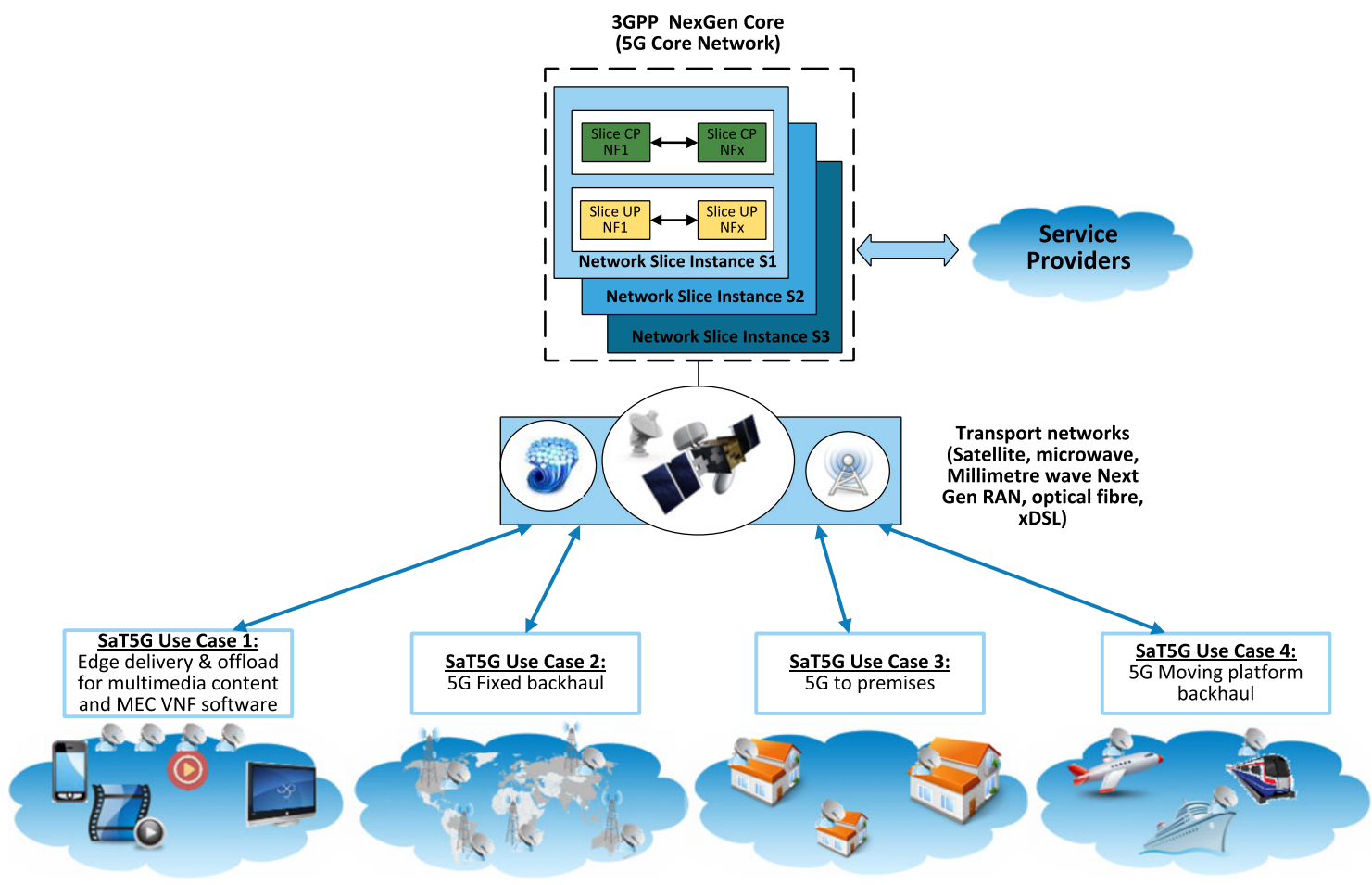

FIGURE 6 SaT5G use cases in 5G integrated satellite-terrestrial networks for enhanced mobile broadband [Colour figure can be viewed at wileyonlinelibrary.com]

satellite link (up to Gbps speed) from geostationary and/or nongeostationary satellites will complement existing terrestrial connectivity, where available. Note that this SUCC assumes that limited or no existing terrestrial connectivity is available. Moreover, the satellite user links are bidirectional since only broadband (ie, unicast, thus VSAT [very small aperture terminal]) communications are supported by this category (ie, no broadcast/multicast). In particular, there is no use of multicasting to populate edge caches in this SUCC, which corresponds to a major difference with respect to the other SUCCs. SaT5G use case 2 "5G fixed backhaul" corresponds to this SUCC in $5 G$.

- Backhauling and tower feed: This SUCC in 5G is about high speed backhaul connectivity to individual cells, with the ability to multicast the same content (eg, video, HD [high definition]/UHD [ultrahigh definition] TV, as well as other nonvideo data) across a large coverage area (eg, for local storage and consumption), as shown in Figure 9 below. The same capability also allows for the efficient backhauling of aggregated IoT traffic from multiple sites. A very high speed, multicast-enabled, satellite link (up to Gbps speed), direct to the local cell towers, from geostationary and/or nongeostationary satellites will complement existing terrestrial connectivity. Note that this SUCC assumes that satellite connectivity will complement existing terrestrial connectivity. Moreover, the satellite user links are either bidirectional and/or unidirectional because, depending on the case, broadband (ie, unicast, thus VSAT terminals) and/or broadcast/multicast (thus, receive only

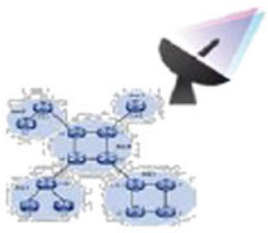

Trunking and Head-End Feed

Satellites provide a very high speed direct connectivity option to remote / hard-toreach locations
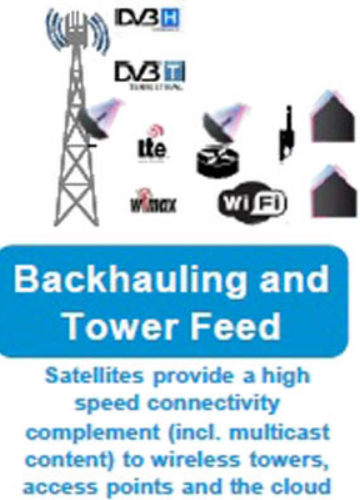

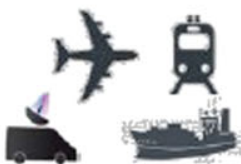

\section{Comms on the move}

Satellites provide a direct and/or complementary connection for users on the move (e.g. on planes, trains, automobiles and ships)

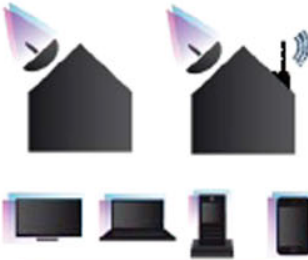

\section{Hybrid Multiplay}

Satellites deliver content complementing terrestrial broadband (as well as direct broadband connectivity in some cases)

FIGURE 7 Satellite use case categories in 5G (or else referred to as satellite "sweet spots" in 5G) [Colour figure can be viewed at wileyonlinelibrary.com] 


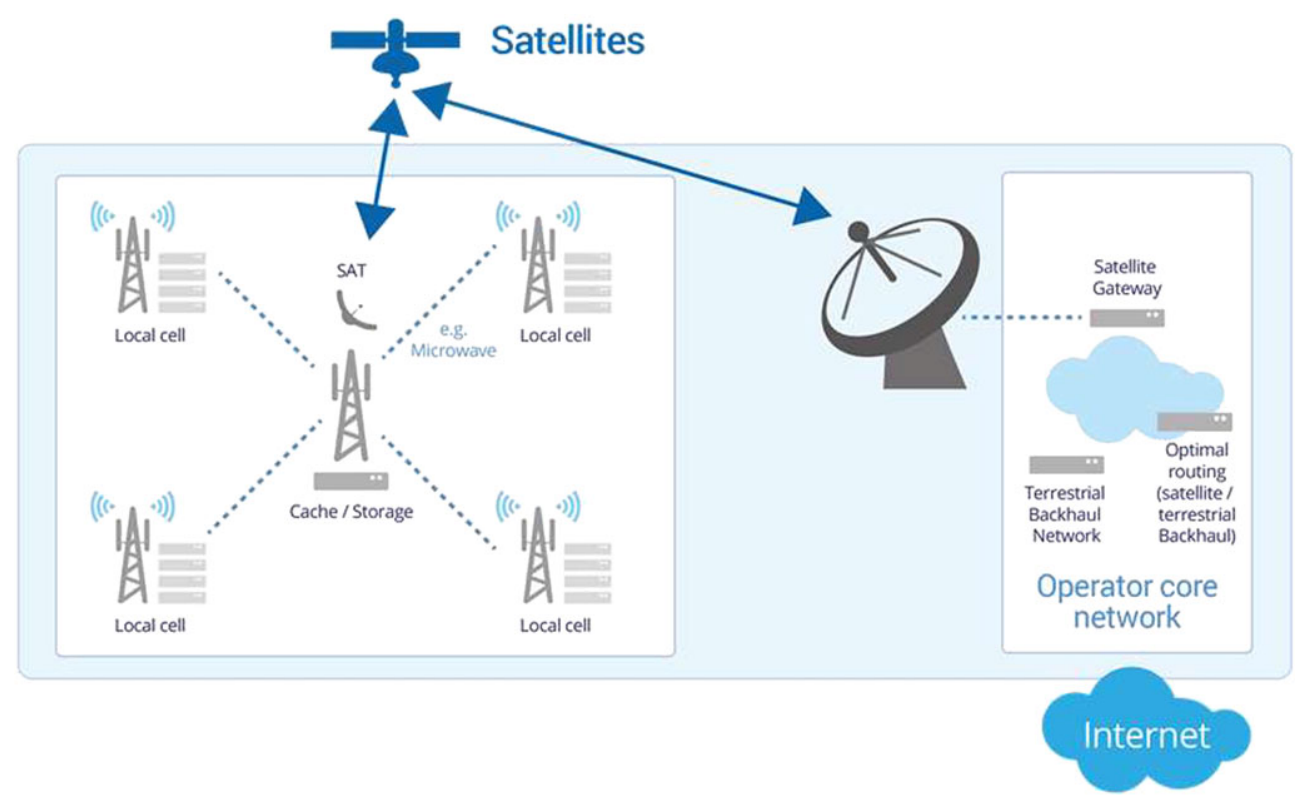

FIGURE 8 Trunking and head-end feed (source: ESOA $^{17}$ ) [Colour figure can be viewed at wileyonlinelibrary.com]

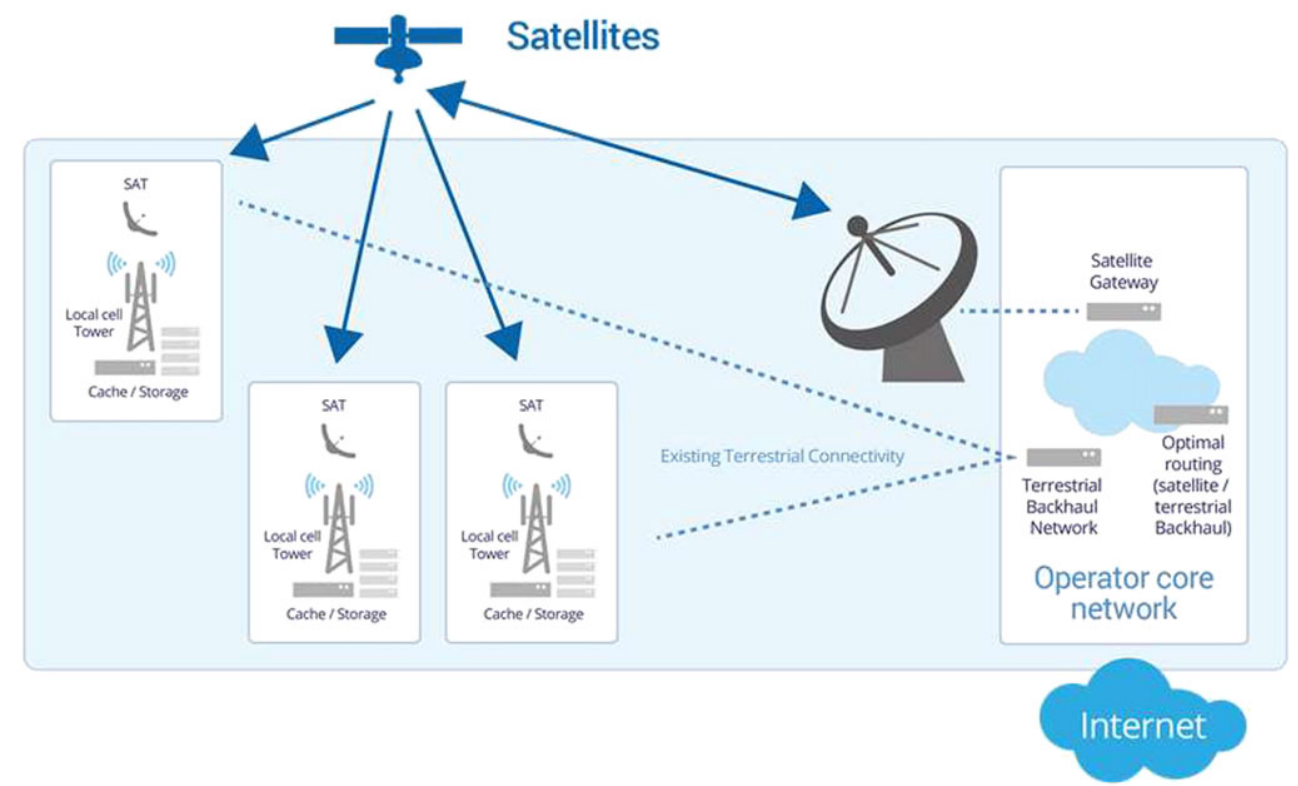

FIGURE 9 Backhauling and tower feed (source: $\mathrm{ESOA}^{17}$ ) [Colour figure can be viewed at wileyonlinelibrary.com]

terminals) communications are supported by this category. In particular, the use of multicasting to populate edge caches is a major difference of this SUCC with respect to the previous one. SaT5G use case 1 "edge delivery and offload for multimedia content and MEC VNF software" corresponds to this SUCC in $5 \mathrm{G}$.

- Communications on the move: This SUCC in 5G is about high-speed backhaul connectivity to individual in-motion terminals on airplanes, vehicles, trains, and vessels (including cruise ships and other passenger vessels), with the ability to multicast the same content (eg, video, HD/UHD TV, software over-the-air, firmware over-the-air, as well as other nonvideo data) across a large coverage area (eg, for local storage and consumption), as shown in Figure 10 below. The same capability also allows for the efficient backhauling of aggregated loT traffic from these moving platforms. A very high speed, multicast-enabled, satellite link (up to Gbps speed), direct to the plane, vehicles, train, or vessel, from geostationary and/or nongeostationary satellites will complement existing terrestrial connectivity, where available. Note that this SUCC assumes that satellite connectivity will complement existing terrestrial connectivity, where available (such as, airports, harbors, train stations, and connected cars). Moreover, the satellite user links are either bidirectional and/or unidirectional because, depending on the case, broadband (ie, unicast, thus VSAT terminals) and/or broadcast/multicast (thus, receive only terminals) communications are supported by this category. SaT5G use case 4 " $5 \mathrm{G}$ moving platform backhaul" corresponds to this SUCC in $5 \mathrm{G}$. 


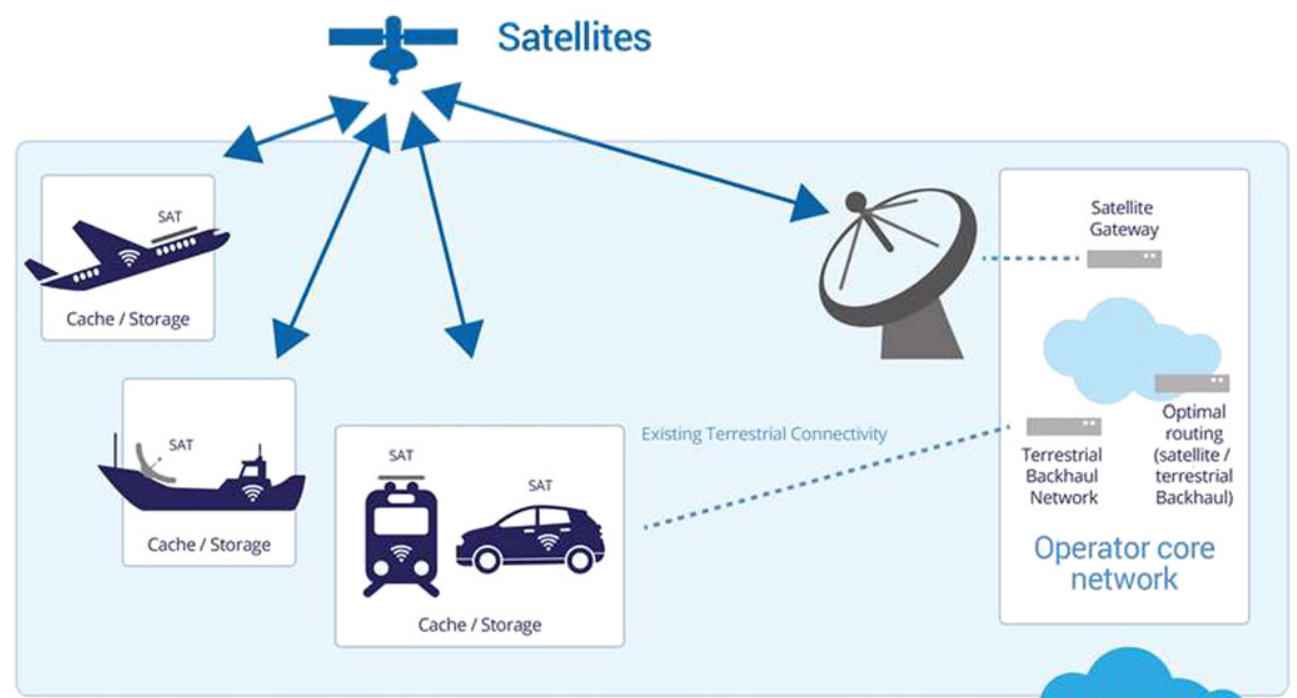

Internet

FIGURE 10 Communications on the move (source: ESOA ${ }^{17}$ ) [Colour figure can be viewed at wileyonlinelibrary.com]

- Hybrid multiplay: This SUCC in 5G is about high-speed connectivity including backhaul to individual homes and offices, referred to as premises, with the ability to multicast the same content (video, HD/UHD TV, and other nonvideo data) across a large coverage area (eg, for local storage and consumption). The same capability also allows for an efficient broadband connectivity for aggregated loT data. In-home distribution via Wi-Fi or home/office small-cell (femtocell) is shown in Figure 11 below. A very high speed, multicast-enabled, satellite link (up to Gbps speed), direct to the home or office, from geostationary and/or nongeostationary satellites will complement existing terrestrial connectivity. Direct-to-home (DTH) satellite TV, integrated within the home or office IP network, will further complement this use case. Note that this SUCC assumes that satellite connectivity will complement existing terrestrial connectivity. Moreover, the satellite user links are either bidirectional and/or unidirectional because, depending on the case, broadband (ie, unicast, thus VSAT terminals) and/or broadcast/multicast (thus, receive only terminals) communications are supported by this category. SaT5G use case 3 " $5 \mathrm{G}$ to premises" corresponds to this SUCC in $5 \mathrm{G}$.

\section{4 | Relevance to SaT5G research pillars}

The selected SaT5G use cases are mapped to the 6 SaT5G RPs as shown in Table 3 below.

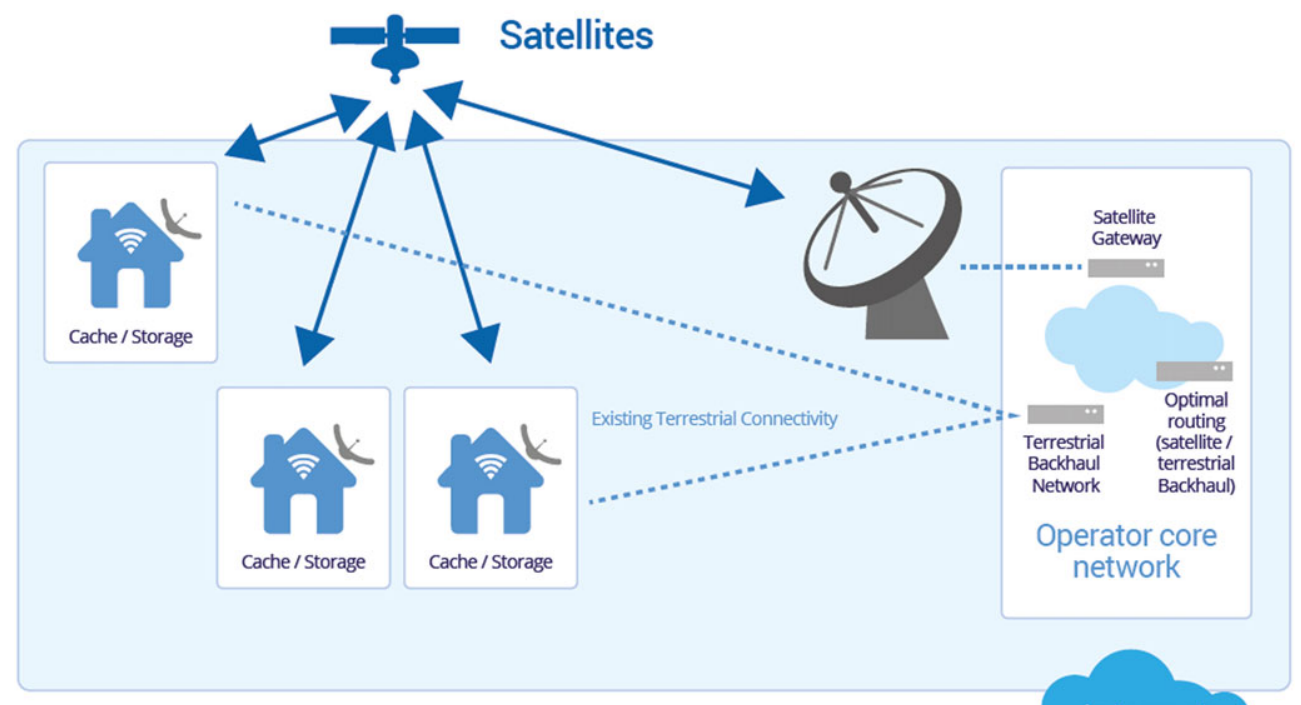

Internet

FIGURE 11 Hybrid multiplay (source: $\mathrm{ESOA}^{17}$ ) [Colour figure can be viewed at wileyonlinelibrary.com] 
TABLE 3 Mapping of SaT5G use cases to SaT5G research pillars

\begin{tabular}{|c|c|c|c|c|}
\hline $\begin{array}{l}\text { Research } \\
\text { Pillar }\end{array}$ & $\begin{array}{l}\text { SaT5G Use Case 1: Edge Delivery and } \\
\text { Offload for Multimedia Content and } \\
\text { MEC VNF Software }\end{array}$ & $\begin{array}{l}\text { SaT5G Use Case 2: } 5 \mathrm{G} \\
\text { Fixed Backhaul }\end{array}$ & $\begin{array}{l}\text { SaT5G Use Case 3: } 5 \mathrm{G} \\
\text { to Premises }\end{array}$ & $\begin{array}{l}\text { SaT5G Use Case 4: } 5 \mathrm{G} \\
\text { Moving Platform Backhaul }\end{array}$ \\
\hline RP I & \multicolumn{4}{|c|}{$\begin{array}{l}\text { Virtualization of satellite functional components and integration of the satellite transport link in the SDN/NFV architecture and support of } \\
\text { network slicing feature }\end{array}$} \\
\hline RP II & $\begin{array}{l}\text { End-to-end service life cycle management } \\
\text { and orchestration which includes virtual } \\
\text { and physical IT and network resources }\end{array}$ & $\begin{array}{l}\text { Integrated } 5 \mathrm{G}-\text { SatCom virtual } \\
\text { and physical resource } \\
\text { orchestration and service } \\
\text { management }\end{array}$ & $\begin{array}{l}\text { Flexible integrated } 5 \mathrm{G}-\text { SatCom } \\
\text { resource } \text { orchestration and } \\
\text { service life cycle } \\
\text { management }\end{array}$ & $\begin{array}{l}\text { Mobility aware end-to-end } \\
\text { service life cycle } \\
\text { management and } \\
\text { resource }^{a} \text { orchestration }\end{array}$ \\
\hline RP IV & Support of multicast traffic & $\begin{array}{l}\text { Support of NG2/NG3 } \\
\text { protocols }\end{array}$ & $\begin{array}{l}\text { Support traffic splitting/link } \\
\text { aggregation solutions }\end{array}$ & $\begin{array}{l}\text { Support of } \mathrm{NG} 2 / \mathrm{NG}^{\mathrm{b}} \\
\text { dynamic relocation }\end{array}$ \\
\hline RP V & $\begin{array}{l}\text { Extension of the security architecture to } \\
\text { broadcast component }\end{array}$ & \multicolumn{2}{|c|}{$\begin{array}{l}\text { Efficient key management and authentication over fixed satellite } \\
\text { transport }\end{array}$} & $\begin{array}{l}\text { Efficient key management } \\
\text { and authentication over } \\
\text { mobile satellite transport }\end{array}$ \\
\hline
\end{tabular}

${ }^{a}$ In the SDN/NFV enabled SaT5G ecosystem, network slices are composed by a wide range of resources, such as IT assets and bandwidth.

${ }^{b} \mathrm{NG} 2$ corresponds to the $5 \mathrm{G}$ control plane interface, whereas NG3 to the $5 \mathrm{G}$ user plane interface.

\section{I SCENARIOS FOR SELECTED SATELLITE USE CASES}

This section provides a qualitative high-level description of the scenarios associated to each selected SaT5G use case. By definition, the scenarios for SaT5G use cases correspond to instantiations of the SaT5G use cases for the accomplishment of a specific duty. As such, a scenario for a SaT5G use case drives the integrated network topology and the architecture design.

These scenarios are summarized in Table 4 below. Due to space limitations, not all the scenarios listed above are presented here. To this end, the interested readership is referred to SaT5G Consortium. ${ }^{25}$

SaT5G focuses primarily on backhaul via satellite. To this end, note that the scenarios for the selected SaT5G use cases drive mainly the design of backhauling architectures where there is satellite connectivity between the $5 \mathrm{G}$ core network and the radio access network (RAN). The architecture design for the given scenarios corresponds to currently ongoing work within the SaT5G project, whose results will be reported in future publications (see eg, Tiomela Jou et $\mathrm{al}^{26,27}$ ). As such, it is beyond the scope of this paper.

\section{1 | Scenarios for Use Case 1: Edge delivery and offload for multimedia content and MEC VNF software}

The scenarios associated to this use case correspond to satellite broadcast/multicast functions and the use of caching. This can be implemented via a standalone fixed terminal or via delivery to the mobile edge cache for onward delivery to UEs within the 5G MNO network.

Adding broadcast/multicast resources in the network to be able to deliver the most popular on demand as well as live content toward the edge nodes of the network enables to offload a significant part of the traffic and/or to optimize the network infrastructure dimensioning (especially the backhaul links) in the lower density populated areas, where the cost per user is the highest.

Satellites are well suited to provide such broadcast/multicast resources over wide areas so as to aggregate the largest audience possible and hence to reduce the global delivery cost. Combining satellite broadcast/multicast resources with the terrestrial unicast resources is a powerful way

TABLE 4 Scenarios for SaT5G use cases

\begin{tabular}{|c|c|}
\hline Selected Satellite Use Cases for eMBB & Scenarios for Selected Satellite Use Cases \\
\hline $\begin{array}{l}\text { Use case 1: edge delivery and offload for multimedia } \\
\text { content and MEC VNF software }\end{array}$ & $\begin{array}{l}\text { Scenario 1a: offline multicasting and caching of video content and VNF software through satellite } \\
\text { links } \\
\text { Scenario 1b: online prefetching of video segments through satellite links }\end{array}$ \\
\hline Use case $2: 5 \mathrm{G}$ fixed backhaul & $\begin{array}{l}\text { Scenario 2a: satellite backhaul to groups of cell towers } \\
\text { Scenario 2b: satellite backhaul to individual cell towers } \\
\text { Scenario 2c: satellite backhaul to individual small cells }\end{array}$ \\
\hline Use case 3 : $5 \mathrm{G}$ to premises & $\begin{array}{l}\text { Scenario 3a: hybrid multiplay (satellite/xDSL) at home/office premises in underserved areas } \\
\text { Scenario 3b: hybrid multiplay (satellite/cellular) at home/office premises in underserved areas }\end{array}$ \\
\hline Use case 4 : $5 \mathrm{G}$ moving platform backhaul & $\begin{array}{l}\text { Scenario 4a: updating content for on-board systems and grouped media request by the moving } \\
\text { platform company } \\
\text { Scenario } 4 \mathrm{~b} \text { : broadband access for passengers and individual media requests } \\
\text { Scenario 4c: business and technical data transfer for the moving platform company }\end{array}$ \\
\hline
\end{tabular}


to optimize the content delivery costs and improve scalability. The $5 \mathrm{G}$ network infrastructure selects the most appropriate resources according to the audience reached. It can convey "VoD" services (pull model), "TV channels," and "Live events" (push model) and optimize the cost in the same way. Moreover, as the audience for a TV channel varies over time, the delivery method can be adapted to optimize the network bandwidth and cost. Delivery of MEC/VNF software updates can also be accommodated but would need to have greater reliability than some other services.

The service and network providers can use the geographic popularity hints to optimize the caching decision process even further and use satellite broadcast to reach the caching nodes of a popular program region directly. This direct satellite delivery also benefits popular live content, as the time-consuming establishment of multicast trees across terrestrial networks can be avoided. Such a hybrid solution of content delivery to $5 \mathrm{G}$ edge nodes via (terrestrial) unicast and (satellite) broadcast/multicast resources will require adjustments to the eNodeB and other equipment as described in the scenarios below.

Operational scenarios embrace either the direct caching at a fixed terminal or caching at the mobile network edge for the MNOs to deliver to UEs as part of the $5 \mathrm{G}$ network. In the network infrastructure that is owned by an MNO, some IT resources (computing and storage) that are located at the network edge (eg, close to eNodeBs) can be virtualized and leased by the MNO's to third parties such as content providers. The content providers can use the virtualized storage and computing resources at the mobile edge to deploy their content and intelligence, eg, local caching, broadcasting, or multicasting of content to selected mobile edges where there are potentially large crowds of consumers on the content. We call such a virtualized mobile edge a virtual CDN (content delivery network) node. Now we consider the following 2 complementary scenarios: (1) with spatiotemporal knowledge of content popularity at different locations, selected content can be broadcasted or multicasted to the targeted mobile edge CDN nodes through satellite links a priori, so that content has already been cached locally by the time the consumers make the requests and (2) a virtualized CDN node can perform online prefetching (through satellite links) of just-in-time video segments during a video session to ensure enhanced video quality E2E. This operation is useful to the video content applications where content is chunked into fixed-length segments (eg, MPEG-DASH [Moving Picture Experts Group-Dynamic Adaptive Streaming over HTTP]). In this scenario, the virtual CDN node does not need any knowledge about content popularity a priori.

\subsection{1 | Scenario 1a: Offline multicasting and caching of video content and VNF software through satellite links}

In case of video content, each virtual CDN node should be capable of monitoring and predicting the popularity of content objects in its local area, and making necessary decisions whether some content should be cached locally from the remote content origin a priori. In case the virtual CDN node has predicted that a specific content is expected to become popular in its region, it can issue a request to the original source to cache the content locally even before the local consumers start to make the requests. For such a purpose, satellite links can play a useful role to offload content traffic away from the terrestrial networks between the original content source and a virtual CDN node. It can be inferred that each virtual CDN node independently performs its own content popularity monitoring and prediction. As such, even multiple virtual CDN nodes predict the same content object to become popular, they may issue the content requests to cache at different times. To maximize the benefit of broadcast and multicast, it is important that a content broadcast/multicast scheduling intelligence is in place such that (1) a (expected-to-be-popular) content object can always be delivered in time to individual virtual CDN nodes for caching before a large number of local users start to make the requests, and (2) the content traffic through the satellite links does not incur any potential congestions due to ad hoc content requests (incurred by the prediction outcome of local content popularity) from individual mobile CDN nodes. Figure 12 below illustrates the top-level operation of such broadcast/multicast operations performed by mobile edge virtual CDN nodes: Popular content A is multicasted through satellite links to the virtual CDN nodes in regions 1 and 2, while popular content B is broadcasted through satellite links to all the 3 regions according to the popularity learning/ prediction outcome performed at the mobile edge.

On the other hand, such multicasting/broadcasting and caching techniques can be also applied for supporting VNF software updating at different sites. Compared with video content caching which requires popularity monitoring and prediction, the distribution task of software updates is more straightforward without complex intelligence. However, considering traffic load dynamicity over the satellite link, delay-tolerant VNF distribution operations can be scheduled during off-peak time (eg, mid-night) when the content traffic load is expected to be on its low level.

\subsection{2 | Scenario $1 \mathrm{~b}$ : Online prefetching of video segments through satellite links}

In recent years, video content providers such as YouTube and Netflix have been adopting the MPEG-DASH standard to provide streaming services. ${ }^{28}$ In this scenario, a video content is chunked into fixed segments which can be independently requested and adapted with multiple quality resolutions. While MPEG-DASH has many benefits such as offering flexibility through on-the-fly quality adaptation and its easy implementation over existing HTTP (Hypertext Transfer Protocol) infrastructure, the fact that DASH uses TCP (Transmission Control Protocol) is a double-edged sword. On the one hand, it means reliable content delivery and that video quality degradation caused by, eg, loss of I-frames can be avoided. On the other hand, when a wireless UE streams a video, there are 2 network segments on the E2E path that have distinctively different characteristics, which are (1) RAN that is wireless and (2) the mobile core network and the public Internet that are typically wired or via satellite links. Specifically, the wired/satellite segment has high bandwidth-delay-product due to the high-capacity links and long latency due to the long data transport distance across the global Internet. The worst-case E2E latency across the terrestrial Internet is around 300 ms, and if satellite links are involved as the backhaul, this latency can be increased to 500+ ms. In contrast, the wireless access segment has much lower bandwidthdelay-product due to limited radio resource capacity over the air interface and relatively lower latency. Transmission Control Protocol does not 


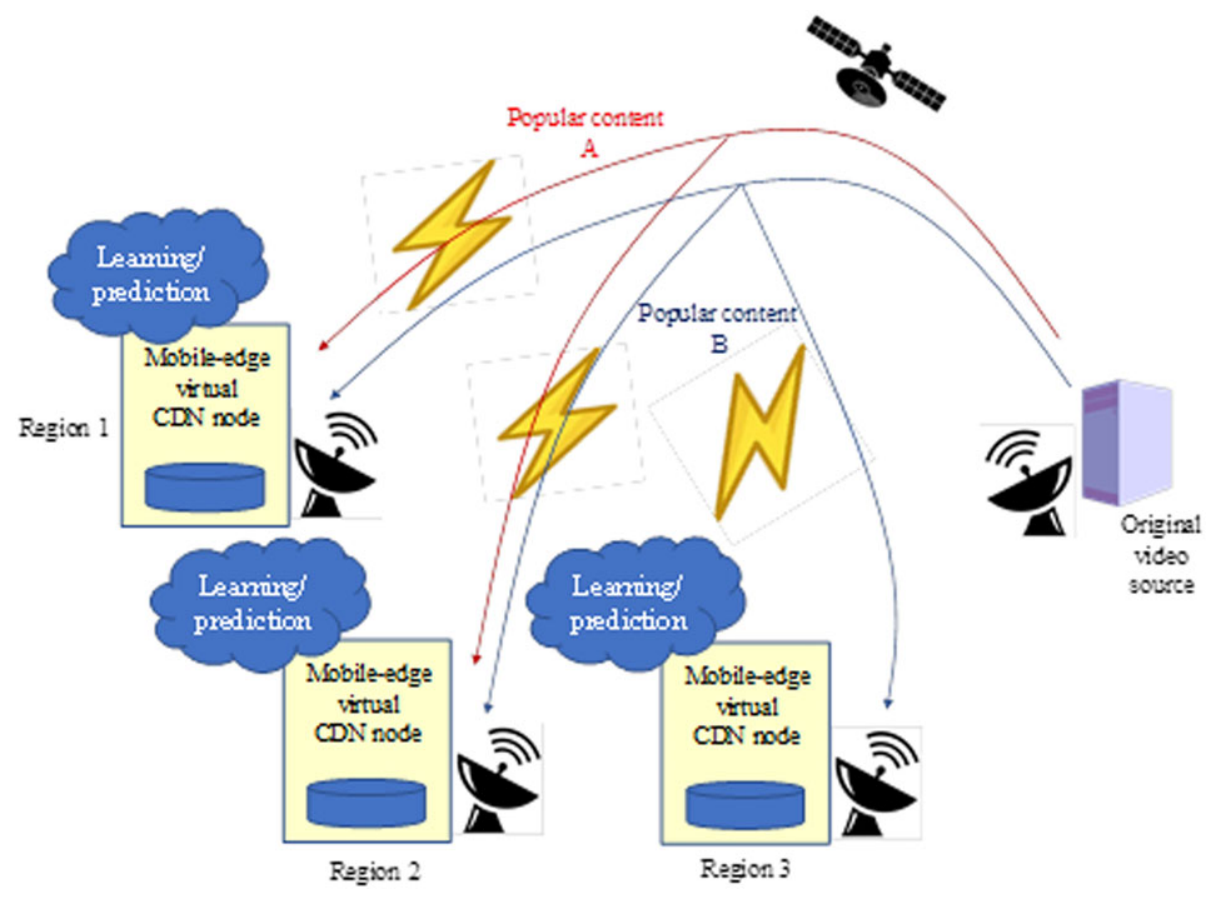

FIGURE 12 Offline broadcast/multicast content objects through satellite links [Colour figure can be viewed at wileyonlinelibrary.com]

perform well on E2E paths consisting of 2 segments with such different characteristics. ${ }^{29}$ Even when there is no RAN resource competition, the Internet is unable to support seamless $4-\mathrm{K}$ video streaming in many scenarios.

Based on this observation, we propose to introduce a novel online video delivery scheme which particularly aims at providing quality of experienceassured 4-K VoD (video on demand) streaming to mobile users at a global Internet scale, even with satellite links involved. The proposed scheme contains the following key operations at the mobile edge virtual CDN node: First, it realizes context awareness on network and users. For network context, it captures the RAN condition that is disseminated by the MNO through the Radio Network Information Service as specified by the ETSI MEC paradigm. Second, it performs adaptive prefetching on a per-user per-session basis; ie, it predownloads video segments from the video source and maintains a progress gap ahead of the user's actual request progress. Such a gap is adaptive and is optimized based on its real-time knowledge on network and user context on-the-fly. Third, it performs video quality adaptation on a per-segment basis also based on its context awareness, such as user mobility pattern, etc.

A high-level overview of the system architecture is presented in Figure 13. From the UE's perspective, the mobile edge is responsible for handling all of its video segment requests during VoD sessions. If a requested video segment is available at the mobile edge, it is served to the UE immediately with low access latency because it is located at the network edge. If it is not available, the mobile edge forwards the request to the original video source, retrieves the requested segment, and serves it to the UE. Furthermore, the mobile edge's embedded content intelligence also performs prefetching by downloading video segments in advance, ie, ahead of the UE's request progress.

\section{2 | Scenarios for Use Case 2: 5G fixed backhaul}

The scenarios associated to this use case correspond to a wide range of scenarios. We have considered specifically the following 3 scenarios based on the relative geographical reach of satellite backhaul.

- Scenario 2a: satellite backhaul to groups of cell towers;

- Scenario $2 b$ : satellite backhaul to individual cell towers; and

- Scenario 2c: satellite backhaul to individual small cells.

In all cases, the user and control plane data are interconnected to the core via the satellite gateway.

Due to space limitations, only Scenario $2 \mathrm{~b}$ is elaborated below, whereas the interested readership in Scenarios $2 \mathrm{a}$ and $2 \mathrm{c}$ is referred to SaT5G Consortium. ${ }^{25}$

\subsection{1 | Scenario 2b: Satellite backhaul to individual cell towers}

This would be satellite backhauls to a cell tower covering a region where there is no cost-effective terrestrial backhaul option. This includes satellite backhaul service to the following:

- rural and remote locations in developed countries; 


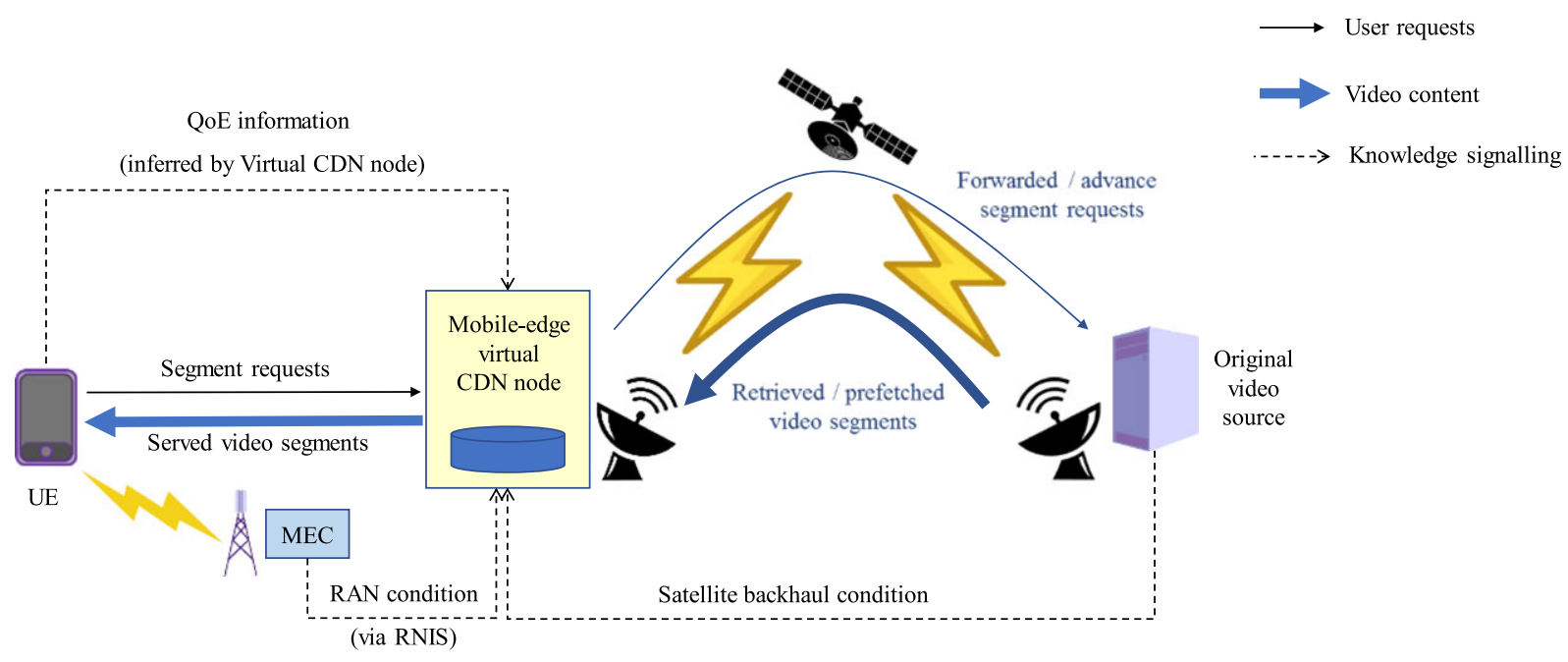

FIGURE 13 Online prefetching of Moving Picture Experts Group-Dynamic Adaptive Streaming video segments through satellite links [Colour figure can be viewed at wileyonlinelibrary.com]

- anywhere outside urban areas in developing countries; and

- islands, mountain regions, and other isolated areas.

While there are fundamental differences in detailed implementation, there are also many similarities. The major difference between developed and developing countries is the ability to pay for service which will tend to impact on (a) the number of devices per community of a given size, (b) the amount of data an eMBB smart phone user can afford, and (c) the methods of payment.

Undoubtedly, such a cell site would also support mMTC communications from loT devices extending smart city like capabilities to such regions (eg, smart villages and in particularly agri-tech functions ${ }^{30}$ ).

In this context, the following representative scenario has been defined:

Scenario $2 \mathrm{~b}$ : Satellite backhaul to a single cell tower located in a rural area in the EU covering 2 villages about $5 \mathrm{~km}$ apart and a rural main road. The villages are home to 300 families; in summer months, an additional 50 families may be in holiday accommodation. The road can occasionally be busy with holiday traffic but is usually quiet. The predominant traffic on the cell is eMBB, but there is some mMTC traffic generated by agri-tech.

\section{3 | Scenarios for Use Case 3: $5 \mathrm{G}$ to premises}

The scenarios associated to this use case is mainly relevant to homes and small office home office premises located in underserved areas of developed countries, which are served with terrestrial telecommunication network infrastructure (xDSL [digital subscriber line] or cellular access) of poor bandwidth performance (eg, users are located far from the DSL access multiplexer or far from 4G cell tower).

In such underserved areas of developed countries, the use of satellite to complement the existing terrestrial broadband access link can lead to a hybrid satellite/terrestrial multiplay scenario which can be envisaged to benefit from low-latency of terrestrial networks and high-bandwidth of satellite networks. In particular, complementing the existing and performance-limited terrestrial broadband link (xDSL or cellular access) by a satellite broadband link with multicast and caching capabilities is considered here.

Similar hybrid satellite/XDSL scenarios have been considered in MENDHOSA ${ }^{9}$ as well as in other ESA studies. ${ }^{31,32}$ This set of scenarios is particularly relevant if satellite can provide more bandwidth for premium clients, typically multiscreen and UHD, and if user experience for Internet applications is raised to a level similar than those of terrestrial networks (latency, throughput at peak hours for a large number of clients, etc). To this end, the use of new generation High Throughput Digital Broadcasting Satellite Systems (or else referred to as hybrid broadband/broadcast satellites ${ }^{24}$ ) is important not only to maintain the satellite opportunity for broadband while bringing the communication cost significantly down but also to further boost the direct broadcast satellite services.

Moreover, much mobile data activity takes place within users' homes. For users with fixed broadband and Wi-Fi access points at home, or for users served by operator-owned femtocells and picocells, a sizable proportion of traffic generated by mobile and portable devices is offloaded from the mobile network onto the fixed network. As estimated in Cisco VNI, ${ }^{33}$ by 2021, 63\% of all traffic from mobile-connected devices (almost 84 exabytes) will be offloaded to the fixed network by means of Wi-Fi devices and femtocells each month.

In this context, high-speed satellite links empowered with multicast and caching capabilities, direct to the home or office, providing the broadcast content and offloading existing terrestrial connectivity are considered here to take DTH a step beyond. The benefits of this set of scenarios are mainly 2-fold: 
- Satellite coverage allows homogeneous service offering-anywhere.

- Multicast and caching enable bandwidth savings and improved quality of service/quality of experience.

To this end, we have considered specifically the following 2 scenarios:

- Scenario 3a: hybrid multiplay (satellite/xDSL) at home/office premises in underserved areas; and

- Scenario 3b: hybrid multiplay (satellite/cellular) at home/office premises in underserved areas.

Due to space limitations, only Scenario $3 a$ is elaborated below, whereas the interested readership in Scenario $3 \mathrm{~b}$ is referred to SaT5G Consortium. ${ }^{25}$

\subsection{1 | Scenario 3a: Hybrid multiplay (satellite/xDSL) at home/office premises in underserved areas}

This Scenario corresponds to a multilink network configuration with xDSL terrestrial link being augmented by the addition of a satellite broadband link with broadcast/multicast and caching capabilities.

It is mainly relevant to homes and small office home office premises located in underserved areas of developed countries, which are served with xDSL links of poor bandwidth performance (eg, users are located far from the DSL access multiplexer). As such, the xDSL link is of such poor quality that it cannot carry any multicast video.

Multiple devices are considered in the home/office environment. The advances in user devices, coupled with innovative services drive user expectations, in particular in choice, quality, availability, and affordability. There is a wide and growing choice of devices which can receive media services for viewing and listening, from stationary TV sets and home radio receivers to personal computers, tablets and smart phones, game consoles, media boxes, or even whole wall UHDTV displays. A rapidly growing number of smart phones' and tablets' displays enable high-quality video, and analysts predict that the use of video on such devices will grow substantially. Thus, DTH satellite TV integrated within the home/office IP network is considered in this scenario. Moreover, in-home/in-office distribution via Wi-Fi or home/office small cell (femtocell) is also considered.

Such multilink network configuration Scenario requires 2 distinct functions as dedicated hardware or software equipment: (a) the home/office gateway located at the home/office premises and (b) Internet gateway located in the core network. Moreover, adjustments to the home/office gateway and Internet gateway in the backbone are needed to be able to split/combine the traffic over the existing terrestrial access link and the satellite link, respectively.

To this end, an intelligent user gateway which aggregates the multiple physical networks at the level of the home/office is integrated within the home/office gateway. The associated challenge here corresponds to the fact that these networks are mostly owned by different commercial entities, often competing with each other, that may have no interest in cooperating and sharing costs (eg, on a common home/office gateway).

The home/office gateway includes caching and storage capabilities. Caching is added to each home/office premise to store locally the broadcasted/multicasted content. Efficient caching management algorithms push and store locally the most popular content optimizing the hit ratio and hence the bandwidth saving. Further details are provided in Section 4.1 above.

The home/office gateway also includes the satellite reception hardware. This concept is commercially appealing as it removes this functionality from the set-top-box (STB) and makes satellite delivery more independent of the main STB in-home. Satellite reception becomes a basic feature of a home, and the satellite delivered services become available on any device (and not just the main TV screen STB as in the past).

It is therefore relevant to study the architecture and protocols that would be needed to provide a more robust satellite transmission scheme to every home/office premises and would abstract the end-devices from the actual physical delivery network.

In this context, new protocol stacks (eg, native IP/Multicast-assisted Adaptive Bitrate), which make the multidevice scenario significantly more attractive than it is today, should be further investigated in the subsequent work. Digital rights management and underlying protocol (eg, DASH, HTTP live streaming, and HTTP smooth streaming) will also have an impact on the home/office gateway design particularly in multiscreen environments. Other critical elements toward the realization of this scenario are caching, efficient caching management schemes, implications of chunked video, seamless blending of services, intelligent routing, network technology convergence, and lowering of costs for implementing certain technologies (chipsets) because they use maximum technical commonality, standard end-device functionality to provide access to all content independently on how it was delivered to that device.

In addition, the emergence of new technologies, such as active electronically steerable beam antennas, will facilitate the bundling of services and the overall use case. Such antennas would for example allow the simultaneous reception of both broadcast/multicast and unicast links streamed at different orbital locations, making a totally optimal use of satellite spectrum and capacity, improving service value proposal while lowering CAPEX/OPEX up to the point where satellite may consider regaining footprints in urban areas.

Figure 14 below illustrates the possible installation configuration of the home/office gateway at user premises.

\subsection{Scenarios for Use Case 4: 5G moving platform backhaul}

The scenarios associated to this use case can be summarized as providing high-speed backhaul connectivity to individual moving terminals on airplanes, vehicles, trains, vessels (including cruise ships and other passenger vessels), or even future driverless cars, with the ability to multicast the 


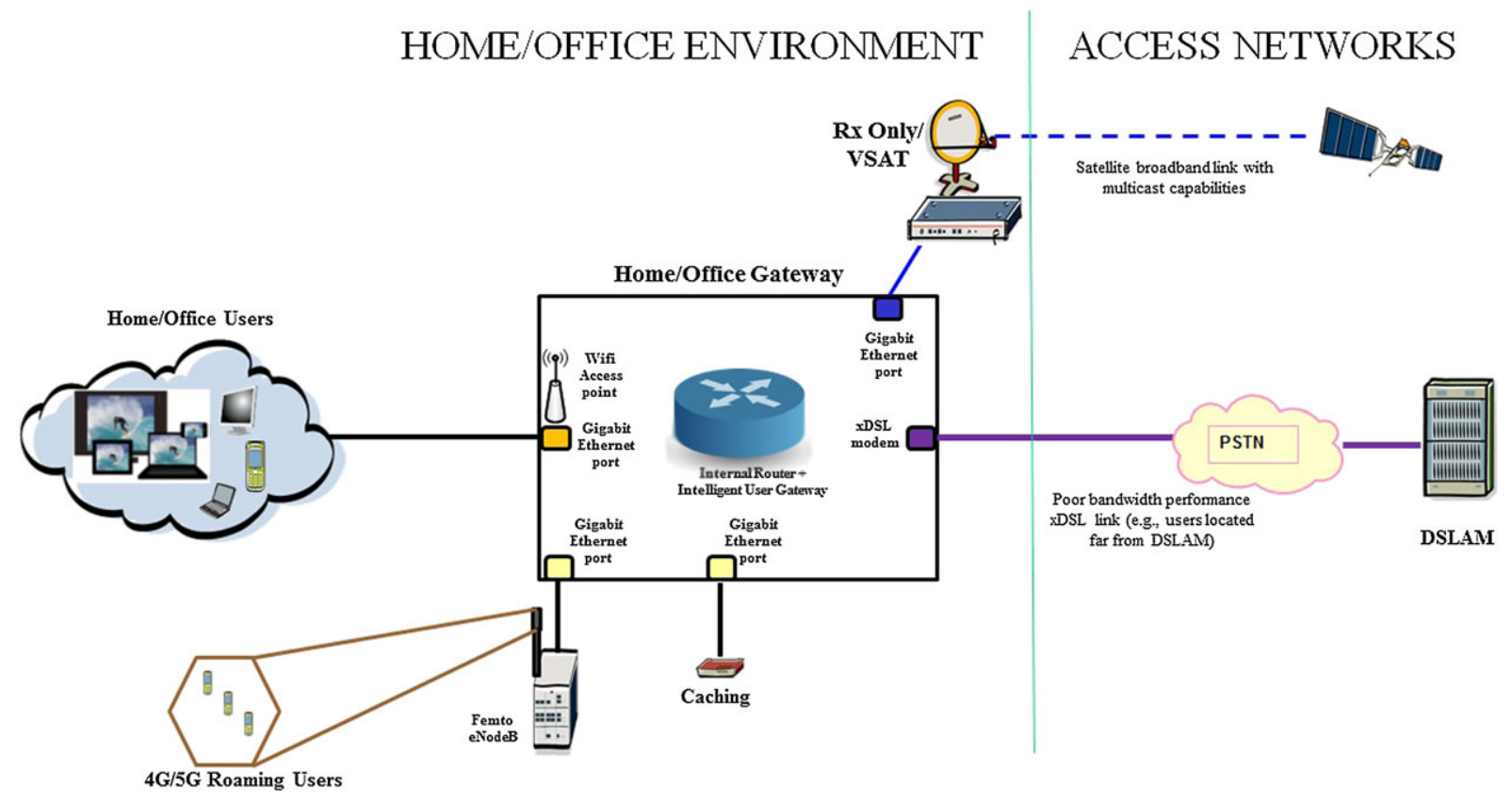

FIGURE 14 Home/office gateway in hybrid multiplay (satellite/xDSL) at home/office premises in underserved areas (source: MENDHOSA, ${ }^{9}$ augmented) [Colour figure can be viewed at wileyonlinelibrary.com]

same content (eg, video, HD/UHD TV, firmware over-the-air, and other nonvideo data) across a large coverage area (eg, for local storage and consumption) and provide efficient broadband access connectivity from/toward these moving platforms.

It should be noted that both satellite stand-alone backhauling and hybrid multiplay, ie, the satellite link acting as a complement of existing terrestrial infrastructure, can be envisaged depending on the scenario and the type of targeted platform.

In this context, we have considered specifically the following 3 scenarios:

- Scenario 4a: updating content for on-board systems and grouped media request by the moving platform company;

- Scenario 4b: broadband access for passengers and individual media requests; and

- Scenario 4c: business and technical data transfer for the moving platform company.

Due to space limitations, only Scenarios $4 a$ and $4 b$ are elaborated below, whereas the interested readership in Scenario $4 c$ is referred to SaT5G Consortium. ${ }^{25}$

\subsubsection{Scenario 4a: Updating content for on-board systems and grouped media request by the moving platform company}

This scenario can be resumed as a grouped request for media by the moving platform company.

This would be a way to update the content proposed by the moving platform company to passengers and subscription to live TV. The end user would use a standalone application on its own device or application preinstalled on the devices provided by the company.

The catalogue is updated with predictive valuable content and most demanded content. Accessible media might include videos, music, game patch, and newspapers. Live TV can also be proposed to broadcast for example live TV show, TV news, or live sport program like a champion's league game.

This scenario is tightly related to caching/multicast edge delivery, exploiting the inherent broadcast capabilities of satellite networks and adding the particularity of being addressed to moving platforms. It is considered of high added value for the moving platform companies being potentially combined with a full broadband access (as described in Scenario 4b).

A stand-alone satellite backhauling can be envisaged for airplanes and vessels (cruise ships and other passenger vessels) and in hybrid mode, complementing existent terrestrial connectivity in trains and other vehicles (buses, trucks, or future driverless cars).

Particularly, in the case of future driverless cars, satellite role would be to provide live broadcast and multicast streams for the passengers when in remote roads, through a phased-array antenna mounted on the rooftop of the car. In that case, a low-capacity moving platform is envisaged because the computing and storage capabilities for MEC, caching, etc functions are expected to be smaller or more costly for a car. 


\subsection{2 | Scenario 4b: Broadband access for passengers and individual media requests}

This scenario proposes a bidirectional broadband access for each passenger for private use, which is transparent to the moving platform. The network requests are therefore individual and proper to each passenger activities.

As in the precedent scenario (Scenario 4a), a stand-alone satellite backhauling can be envisaged for airplanes and vessels (cruise ships and other passenger vessels) and in hybrid mode, complementing existent terrestrial connectivity in trains and other vehicles (buses, trucks, or future driverless cars).

Particularly, in the case of future driverless cars, as in the precedent scenario (Scenario 4a), a low-capacity moving platform is envisaged here as well. The difference here corresponds to the satellite role which would be to provide $5 \mathrm{G}$ broadband access for the passengers when in remote roads.

The passenger would use their own device(s) and the whole applications installed as they do on the ground.

\section{5 | CONCLUSIONS}

This paper presented initial results available from the SaT5G project. ${ }^{1,25}$

It described the concept, objectives, challenges, and RPs addressed by the SaT5G project. It also defined how satellite can be seamlessly integrated into the eMBB usage scenario for 5G, by elaborating on the SaT5G use cases and scenarios for satellite positioning in eMBB.

Specifically, the selected satellite use cases for eMBB addressed in this paper are as follows:

- Use case 1: edge delivery and offload for multimedia content and MEC VNF software: providing efficient multicast/broadcast delivery to network edges for content such as live broadcasts, ad-hoc broadcast/multicast streams, group communications, and MEC VNF update distribution;

- Use case 2: 5G fixed backhaul: Broadband connectivity where it is difficult or not (yet) possible to deploy terrestrial connections to towers, for example, maritime services, coverage on lakes, islands, mountains, rural areas, isolated areas, or other areas that are best or only covered by satellites across a wide geographic region;

- Use case 3: 5G to premises: connectivity complementing terrestrial networks, such as broadband connectivity to home/office small cell in underserved areas in combination with terrestrial wireless or wireline; and

- Use case 4: $5 \mathrm{G}$ moving platform backhaul: broadband connectivity to platforms on the move, such as airplanes or vessels.

For each of the SaT5G use cases above, a set of scenarios have been defined which drive the integrated network topology and the architecture design. Specifically, the subset of scenarios for the selected satellite use cases for eMBB elaborated in this paper is the following:

- Scenarios for use case 1: (1a) offline multicasting and caching of video content and VNF software through satellite links and (1b) online prefetching of video segments through satellite links;

- Scenario for use case 2: (2b) satellite backhaul to individual cell towers;

- Scenario for use case 3: (3a) hybrid multiplay (satellite/xDSL) at home/office premises in underserved areas; and

- Scenarios for use case 4: (4a) updating content for on-board systems and grouped media request by the moving platform company and (4b) broadband access for passengers and individual media requests.

Further work on the requirements definition, business modeling, system architecture definition, research to prototype implementation, validation, and demonstration of the selected satellite use cases and scenarios for eMBB correspond to currently ongoing and future work within the SaT5G project, whose results will be reported in future publications.

\section{ACKNOWLEDGEMENTS}

The work presented in this paper has been conducted as part of the SaT5G (Satellite and Terrestrial Network for 5G) project, which has received funding from the European Union's Horizon 2020 research and innovation program under grant agreement no. 761413. The authors would like to thank their SaT5G consortium partners.

\section{ORCID}

Konstantinos Liolis (D) http://orcid.org/0000-0003-2337-8148

\section{REFERENCES}

1. European Commission H2020 5G PPP project "SaT5G" (Satellite and Terrestrial Network for 5G). 2017. [Online]. Available: http://sat5g-project.eu/. 
2. European Parliament. January 2016. [Online]. Available: http://www.europarl.europa.eu/RegData/etudes/BRIE/2016/573892/EPRS_BRI(2016) 573892_EN.pdf.

3. ITU-R. IMT vision-framework and overall objectives of the future deployment of IMT for 2020 and beyond. [Online]. Available: https://www.itu.int/ dms_pubrec/itu-r/rec/m/R-REC-M.2083-0-201509-I!!PDF-E.pdf.

4. 5G PPP. [Online]. Available: https://5g-ppp.eu/. [Accessed July 2017].

5. European Commission. H2020 ICT-07-2017 call. [Online]. Available: http://ec.europa.eu/research/participants/portal/desktop/en/opportunities/ h2020/topics/ict-07-2017.html. Accessed July 2017

6. European Commission. 5G for Europe: an action plan. European Commission, 14 September 2016. [Online]. Available: http://ec.europa.eu/newsroom/ dae/document.cfm?doc_id=17131.

7. NetWorld2020 SatCom WG. [Online]. Available: http://www.networld2020.eu/satcom-wg/. [Accessed October 2016].

8. ESA, Avanti (Prime Contractor). SPECSI (Strategic Positioning of the European and Canadian Satcom Industry). [Online]. Available: https://artes.esa.int/ projects/specsi. [Accessed July 2017].

9. ESA, Thales Alenia Space France (Prime Contractor). MENDHOSA (Media \& ENtertainment Delivery over Hetnet with Optimized Satellite Architecture). [Online]. Available: https://artes.esa.int/projects/mendhosa. [Accessed July 2017].

10. ESA, Eurescom (Prime Contractor). INSTINCT (scenarios for integration of satellite components in future networks). [Online]. Available: https://artes. esa.int/projects/instinct. [Accessed July 2017].

11. INSTINCT Whitepaper. September 2016. [Online]. Available: https://artes.esa.int/news/new-artes-funded-whitepaper-looks-role-satcoms-5g-context.

12. ESA, Space Hellas (Prime Contractor). CloudSat (scenarios for integration of satellite components in future networks). [Online]. Available: https://artes. esa.int/projects/cloudsat. [Accessed July 2017].

13. SANSA Consortium. SANSA (Shared Access Terrestrial-Satellite Backhaul Network enabled by Smart Antennas). [Online]. Available: http://www.sansah2020.eu/. Accessed July 2017

14. VITAL Consortium. VITAL (VIrtualized hybrid satellite-TerrestriAl systems for resilient and fLexible future networks). [Online]. Available: http://www. ict-vital.eu. Accessed July 2017

15. RIFE Consortium. RIFE (architecture for an Internet for everybody). [Online]. Available: https://rife-project.eu. Accessed July 2017

16. ESA, Nomor Research (Prime Contractor). SCORSESE (the role of satellite in collaborative adaptive bitrate streaming services). [Online]. Available: https://artes.esa.int/projects/scorsese. [Accessed July 2017].

17. ESOA 5G White Paper. Satellite communications services: an integral part of the $5 \mathrm{G}$ ecosystem. ESOA, 2018. [Online]. Available: https://esoa.net/cmsdata/positions/1693\%20ESOA\%205G\%2016pp\%20Booklet\%20Amends\%20SCREEN\%20Final\%201.pdf.

18. SaT5G Consortium. Deliverable D6.2 "standardisation action plan". October 2017.

19. 3GPP SA1. TR 22.891 V14.2.0 (2016-09), feasibility study on new services and markets technology enablers, stage 1. 2016.

20. 3GPP SA1. SMARTER technical reports. 23 July 2016. [Online]. Available: http://www.3gpp.org/news-events/3gpp-news/1786-5g_reqs_sa1.

21. 3GPP SA1. TR 22.863 V14.1.0 (2016-09), feasibility study on new services and markets technology enablers-enhanced mobile broadband; stage 1. 2016.

22. 3GPP SA1. 3GPP TR 22.864 V14.1.0 (2016-09), feasibility study on new services and markets technology enablers-network operation. 2016.

23. 3GPP RAN1. 3GPP TR 38.811V0.1.0 (2017-06), "Study on new radio (NR) to support non terrestrial networks (release 15$)$ ). 2017.

24. ESA, SES (Prime Contractor). HTS-DBS (High Throughput Digital Broadcasting Satellite Systems). [Online]. Available: https://artes.esa.int/funding/highthroughput-digital-broadcasting-satellite-systems-artes-51-3a073-0. [Accessed July 2017].

25. SaT5G Consortium. Deliverable D2.1 "Satellite reference use cases and scenarios for eMBB". August 2017.

26. Tiomela Jou B, Vidal O, Arnal F, Houssin J-M, Chuberre N, Liolis K, Cahill J, Khalili H, Sayyad Khodashenas P, Boutin M, Chau D-K, Sendra Diaz S. Satellite integration into $5 \mathrm{G}$ system for enhanced mobile broadband. IEEE Network Magazine, Special Issue "Integration of Satellite and 5G Networks", 2018 (submitted, currently under review).

27. Tiomela Jou B, Vidal O, Arnal F, Houssin J-M, Liolis K, Cahill J, Khalili H, Sayyad Khodashenas P, Boutin M, Chau D-K, Sendra Diaz S. Architecture options for satellite integration into 5G networks. in EuCNC 2018, Ljubliana, Slovenia, June 2018 (accepted for oral presentation).

28. ISO/IEC JTC 1/SC 29. ISO/IEC 23009-1:2014, information technology-dynamic adaptive streaming over HTTP (DASH)-part 1: media presentation description and segment formats. May 2015. [Online]. Available: https://www.iso.org/standard/65274.html.

29. Ge C, Wang N, Foster G, Wilson M. Towards QoE-assured 4K video-on-demand delivery through mobile edge virtualization with adaptive prefetching. IEEE Trans Multimed. 2017;19(10):2222-2237.

30. IoTONE. IOTONE "Accelerating the Industrial Internet of Things". [Online]. Available: https://www.iotone.com/guide/internet-of-things-designingsmart-villages/g546. [Accessed 2017].

31. ESA, Forsway (Prime Contractor). Satellite extension of xDSL copper wire based networks. [Online]. Available: https://artes.esa.int/projects/satelliteextension-xdsl-copper-wire-based-networks. [Accessed 2017].

32. ESA, Intecs (Prime Contractor). SAT4NET: analysis of satellite downstream boost for xDSL networks. [Online]. Available: https://artes.esa.int/projects/ sat4net. [Accessed 2017].

33. Cisco. Cisco visual networking index: Global Mobile Data Traffic Forecast Update, 2016-2021. Cisco Visual Networking Index (VNI). February 2017. 


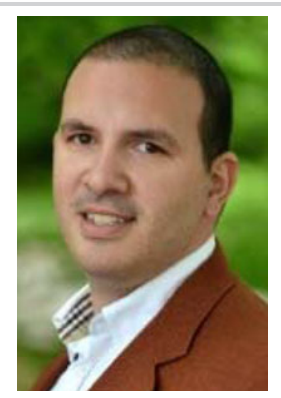

Dr Konstantinos P. Liolis was born in Athens, Greece, in 1981. He received the Dipl.-Eng. degree in Electrical and Computer Engineering from the National Technical University of Athens (NTUA), Greece, in 2004; the MSc degree in Electrical Engineering from the University of California at San Diego (UCSD), USA, in 2006; and the PhD degree in Electrical Engineering from NTUA in 2011. From 2004 to 2006, he was Research Assistant at the California Institute for Telecommunications and Information Technology (Cal-IT2), San Diego, USA. From 2006 to 2008, he was Communication Systems Engineer at the European Space Agency, Research and Technology Centre (ESA/ ESTEC), the Netherlands. From 2008 to 2012, he was R\&D Project Manager at Space Hellas S.A., Greece. Since 2012, he has been with SES S.A., Luxembourg, where he is currently Senior Systems Engineer managing technology innovation projects. He has published more than 60 scientific papers in international peer-reviewed journals, conference proceedings and book chapters, in areas mainly related to satellite communications. He has also numerous contributions to international standardization bodies (ETSI, 3GPP, DVB, ITU-R), and has actively participated in more than 35 R\&D and Innovation projects funded by EU, ESA and National programs on ICT, Space and Security technologies. Dr. Liolis is PMI PMP® certified project manager and is a Member of the Technical Chamber of Greece (TEE). He has served as TPC Member for several international conferences and as Reviewer for several major international journals and conferences. He received a Best Student Paper Award in IEEE RAWCON 2006 and is listed in the "Who's Who in the World"-Edition 2010. From 2009 to 2011, he was elected Chairman of the Future Internet Working Group of ISI, the FP7 ICT European Technology Platform for Satellite Communications.

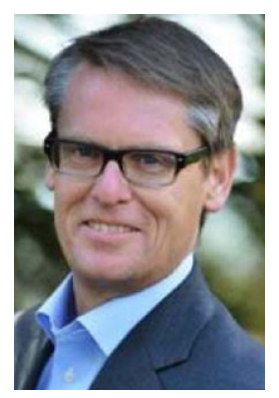

Dr Alexander Geurtz is VP, Strategic Business Innovation at SES. He has over 20 years of experience in satellite and mobile communications from the strategic, business, and technical perspectives. He was a key member of the team that created Solaris Mobile Ltd, an integrated satellite/terrestrial operator in S-Band, representing the company inter alia at 3GPP. He is currently actively involved in SES's business development activities related to the satellite role in 5G ecosystem, incl. 5P PPP, 5G IA, NetWorld2020 ETP, and ESA "Satellite for 5G" Task Force related activities. He received an MS in Electrical Engineering from Delft University of Technology and a PhD from the EPFL in Lausanne, Switzerland.

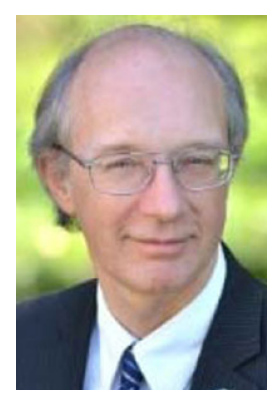

Ray Sperber is a Senior Manager in SES's Systems Engineering department, involved in most SES space segment projects. He has 40 years of experience in satellite communications in various roles mostly having to do with the space segment. From 1991 to 2008, he was the Chief Spacecraft Engineer at SES. He was Avanti Communications' Director of Space Segment from February 2008 through August 2010, giving a keen understanding of broadband. He received a BS in Physics in 1976 and an MS in Aeronautics \& Astronautics 1978, both from MIT, and an MBA from Sacred Heart Luxembourg in 1997. He is a member of IEEE, AIAA, OSA, ASA, BIS \& Da Vinci asbl.

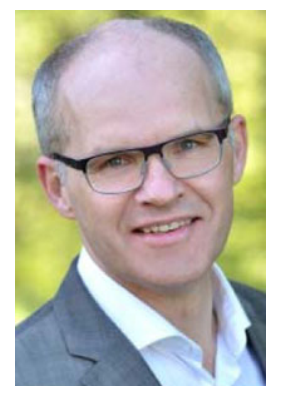

Detlef Schulz was born in Wolfenbuettel, Germany, in 1959. He received his Dipl.-Ing. degree in Telecommunications from the "Fachhochschule Braunschweig/Wolfenbuettel" (Germany) in 1984 and completed the Young Managers Programme of INSEAD (Fontainebleau, France) in 1995. In 1984, he joined FUBA/Hans Kolbe \& Co. (Germany) as Product Manager for commercial and consumer satellite ground segment equipment, VHF/UHF broadcast transmission, reception and signal distribution systems, CATV equipment, and mobile communications. Since he joined SES in Luxembourg in January 1991, he led engineering teams in charge of technical studies, engineering analysis, concept and systems engineering, system tests, recommendations, specifications, standardization, and end-to-end technical solutions for various satellite applications. This included the development of innovative concepts, products and solutions for transmission, reception and networking of video, audio and data signals and the provision of technical guidance to other SES functions, and the industry on the development of corresponding systems and components. Currently, he is Vice President, Concept Engineering, being in charge of overseeing and performing technical studies, concept engineering, technical recommendations and specifications, and technical outlines of end-to-end solutions for enhanced communications systems and advanced satellite systems. These activities and projects have a mid to long term perspective and also consider systems beyond satellite communications as well as competing or complementary transmission infrastructure means. Mr Schulz is also SES's representative in the Steering Board of NetWorld2020, the European Technology Platform for communications networks and services, in the Steering Board and Technical Module of the Digital Video Broadcasting (DVB) project since 1993 and is an invited telecommunication lecturer and member of the Advisory Board of SpaceTech, a Life Long Learning program at the Graz University of Technology (Austria). 


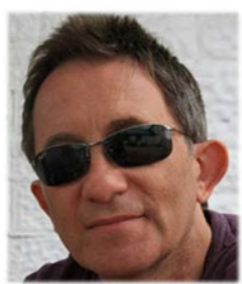

Simon M. Watts has worked in the telecommunications industry since graduation from Manchester University with a BSc in Physics in 1981, with the majority of his experience gained from within the satellite communications industry. Simon has extensive experience of satellite communications and network design having overseen deployment of major satellite networks for BT International and Hughes Network Systems. Simon has worked with Avanti in a Principle Consultant role from early 2012 on multiple ESA, UK, FP7, and $\mathrm{H} 2020$ projects. He has brought wide experience in the telecommunications and broadband network industries at a technical and commercial level to ensure technical and commercial direction. These projects have covered areas including the integration of satellite and terrestrial networks, future satellite networks, modem/antenna design, cellular and Wi-Fi backhaul, mobility, and the network design for incubator services projects in East Africa offering Wi-Fi and multicast content delivery. He was Hughes Network Systems Europe-Chief Engineer-between 2001 and 2011 responsible for designing multiple networks to deliver customer requirements for BP and Camelot UK, as well as the definition of the integrity of all network services and solutions across Europe and the Middle-East. These networks included both significant satellite and terrestrial communication components providing both network connectivity and wide are computer networks using content delivery to run digital signage and training video networks. This role also included the design of all service product solutions including a suite of Internet Broadband services sold in the Middle-East through resellers. Prior to this, he worked at HNS Europe in multiple other roles between 1996 and 2001. His career started at BT International initially helping to design and implement ground station systems before providing VSAT systems in the North Sea for Oil Companies and a VSAT network in southern Africa for a major UK bank. In addition designing digital line of sight and troposcatter systems for deployment in the North Sea for Oil Companies. Simon regularly presents and participates in discussion panels and has supported multiple technical papers whilst at Avanti. He is an MIET.

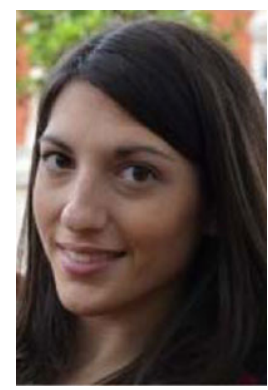

Georgia Poziopoulou is currently working as a Project Manager in Avanti Government Solutions coordinating the 5G-PPP Phase 2 project SaT5G. She joined Avanti in 2013 as a Graduate Engineer and has since worked in Government Solutions making technical contributions to R\&D projects, such as the ESA xDSL project and H2020 SANSA project, as well as supporting new bids. She has also completed a placement in the Satellite Resource Management team performing link budget analyses and supporting everyday operations. She holds an MSc in Wireless and Optical Communications from University College London from which she graduated in 2013 with Distinction and a MEng in Electrical and Computer Engineering from the National Technical University of Athens in Greece. The research projects she has completed during her university studies were focused on power efficiency of linear precoding in MIMO systems and energy efficient communication in Wireless Sensor Networks with the use of time-scheduling and clustering algorithms.

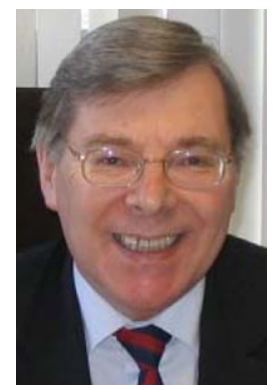

Professor Evans has BSc and PhD degrees from the University of Leeds, is a Fellow of the UK Royal Academy of Engineering, and of the IET and senior member of IEEE and AIAA. From 1968 to 1983, he was British Telecom lecturer to Reader at the University of Essex in Telecommunication systems. He was appointed to the Alec Harley Reeves chair of Information systems engineering at the University of Surrey in 1983 and was founder Director of the Centre for satellite engineering research and then the Centre for Communication Systems Research. He was Dean of Engineering 99-01 and Pro-Vice Chancellor for research and Enterprise from 01-09. Barry Evans has researched in satellite communications, radio propagation, signal processing, and networking and has over 600 publications in the literature plus 3 books. He is Editor of the International Journal of Satellite Communications.

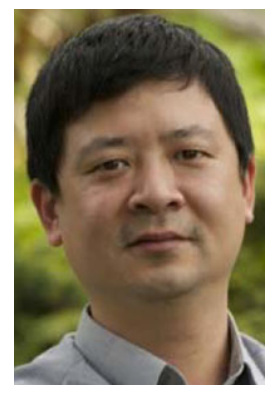

Ning Wang is a Reader at the Institute for Communication Systems (5G Innovation Centre), University of Surrey, UK. He received his B.Eng (Honours) degree from the Changchun University of Science and Technology, P.R. China, in 1996, his M.Eng degree from Nanyang University, Singapore, in 2000, and his PhD degree from the University of Surrey in 2004. His research interests mainly include 5G networking, mobile content delivery, QoS/QoE, and network resource management. 


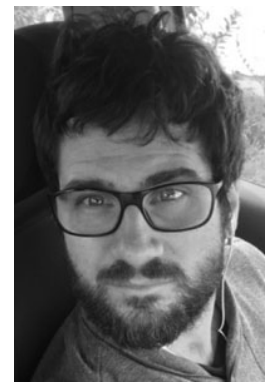

Oriol Vidal Barba was born in Terrassa, Barcelona (Spain), in 1982. He received the Dipl.-Eng. degree in Telecommunications Engineering from the Universitat Autonoma de Barcelona (UAB) in 2008 and the industrial PhD degree in Satellite Communication systems within Airbus Defense and Space in collaboration with ISAE and Université de Toulouse, Toulouse, in 2014. From 2008 to 2011, he was Research Assistant at ISAE, Toulouse, France. From 2014 to mid-2017, he was HTS/VHTS Systems Engineer at Airbus Defence and Space, Toulouse, within the Telecom Business unit. He has been involved and managed several R\&D and Innovation projects funded by EC, ESA, and French National agency (CNES). Since 2017, he holds a Senior System Engineer position and is Head of the Broadband/Broadcast systems team in the Telecom System advanced studies and projects Department.

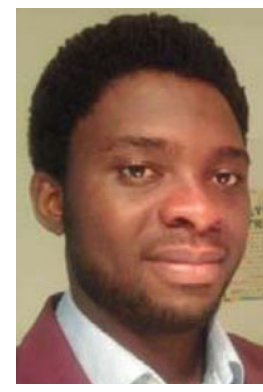

Boris Tiomela Jou was born in Nkongsamba, Cameroon in 1989. After a Bachelor degree in Multinational School of Telecommunications (ESMT) in Yaoundé, Cameroon, in 2009, he received a Master of Engineering in University of Toulouse. From 2011 to 2016, he worked for Thales Alenia Space in Toulouse, France, subsequently as junior engineer in the telecom system department and as satellite modem design engineer in the ground segment department. Since 2016, he has been working for Airbus Defence \& Space in Toulouse, France. In his current position there, within the Telecom System advanced studies and projects Department, he works on various R\&D studies and his developed expertise includes signal processing, network architecture definition and sizing of very high throughput telecoms systems. He has led Airbus Defence \& Space participation to some R\&D projects funded by EU, ESA, CNES, etc. He has been involved in contributions to ETSI, DVB and 3GPP and has co-signed several papers on the satellite communications domain.

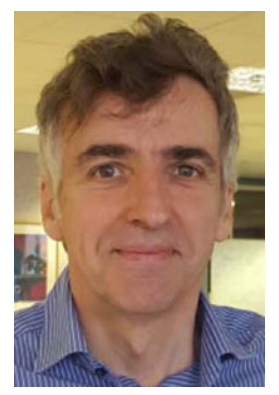

Michael Fitch works in BT Research and Innovation, providing technical leadership to a small research team specialising in physical and systems aspects of wireless communications. He is currently working on a number of projects on emerging wireless technologies such as small cells, radio resource management and 5G. In addition, he provides engineering consultancy to other parts of BT on LTE, WiFi, and other wireless topics. Previous experience is with modelling, trials and deployments of Satellite, WiMAX, 3G, and LTE systems. Michael has a first degree in maths and physics, a PhD in satellite communications and he is a member of the IET.

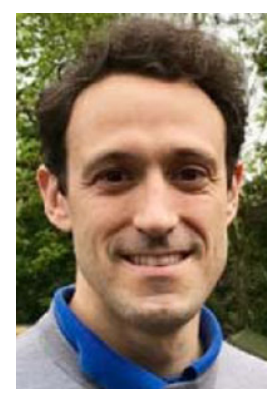

Salva Diaz Sendra was born in Valencia, Spain, in 1980. He received the Tech. Eng. degree in Telecommunication from the Polytechnic University of Valencia (UPV), Spain, in 2003; the Eng. Telecommunications degree from UPV in 2006; the Master in Mobile Telecommunications from UPV and Vodafone Foundation in 2007; the Master in Communications from UPV in 2012; and coursing the PhD from UPV until 2017. From 2005 to 2006, he was a Master Thesis Student at Telenor SA, Trondheim, Norway. In 2007, he was a junior SW developer in GFT, Valencia, Spain. From 2008 to 2012, he was a wireless senior researcher at Mobile Telecommunications Institute at UPV, Valencia, Spain. From 2012 until 2015, he was a project manager in Sistelbanda, Valencia, Spain. Since 2016, he has been with British Telecommunications (BT) PCL, Ipswich, UK, where he is currently a Senior Researcher contributing to 3GPP RAN1 and managing collaborative projects.

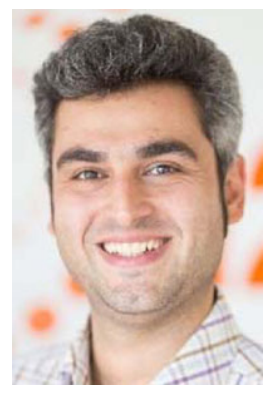

Dr Pouria Sayyad Khodashenas finished the Electronics Engineering B.Sc. in the state University of Guilan, Iran, in 2005. He graduated as the first honered M.Sc. student in Opto-electronic Engineering from the state University of Tabriz, Iran, in 2008. In 2014, he defended his PhD in Optical Telecommunications at Universitat Politécnica de Catalunya (UPC), Barcelona, Spain. His PhD thesis has been distingused as the best UPC PhD thesis in academic year 2013 to 2014. He joined Athens Information Technology (AIT), Athens, Greece, in the same year where he collaborated in the several EU funded projects: FP7 FOX-C, FP7 INSPACE and H2020 ACINO. Since October 2015, he is working as a senior technical lead, solution architect, and project manager at i2Cat Foundation, Barcelona, Spain, collaborating in +10 FP7/H2020 5GPPP projects including FP7 DOLFIN, H2020 SESAME, H2020 SaT5G, H2020 FLAME. Pouria is author/co-author of scientific publications among all, 2 books and 3 book chapters, ICT technical reports and 5GPPP whitepapers, 50+ peer-reviewed articles in the international journals and major conferences. His current research interests include communication systems, 5G core, network architecture and protocols, MANO framework, network function virtualization, software defined networking, resource slicing, marketplace, and brokerage, loT, QoS, techno-economic analysis. 


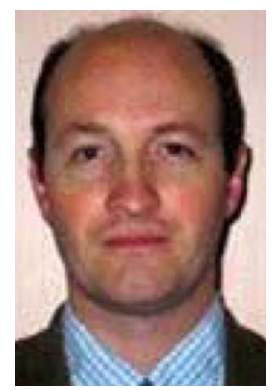

Nicolas Chuberre has more than 25 years of experience in digital radio communications domain including design of cellular handsets/systems (GSM, DECT), management of payload equipment development. He is currently System Engineering Manager, responsible for the design of GSO and Non GSO (Geostationary Synchronous Orbit) satellite communication solutions architecture. He also manages R\&D projects in the satellite communication domain. Nicolas has successfully managed R\&D projects in CNES, ESA, and European Commission context. In particular, he successfully coordinated the 9M€ integrated project MAESTRO (FP6 IST) that led to the launch of an innovative satellite system. Recently, he led an ESA study (Mendhosa) on the role of satellite systems in 5G. Nicolas chairs the Satellite Communication and Navigation working group (SCN WG) of ETSI, the prestandards satcom subgroup of the NetWorld2020 technology platform and vice-chairs the Technical Committee Satellite Earth Stations and Systems of ETSI (TC-SES). He is now an active delegate in 3GPP groups especially in RAN WGs. He has been member of the partnership board of the "5G infrastructure association" during its first three years. Nicolas is the Technical Manager of the EU H2020 5G PPP project SaT5G and leads the standardisation activities.

How to cite this article: Liolis K, Geurtz A, Sperber R, et al. Use cases and scenarios of $5 \mathrm{G}$ integrated satellite-terrestrial networks for enhanced mobile broadband: The SaT5G approach. Int J Satell Commun Network. 2018;1-22. https://doi.org/10.1002/sat.1245 